\title{
可见光催化偕二氟烯烃碳-氟键官能化反应的研究进展
}

\author{
李志清 $b \quad$ 邱潇杨 $b \quad$ 娄 $\quad$ 江 $*, a, b \quad$ 王 强*,a \\ ( ${ }^{a}$ 齐鲁工业大学(山东省科学院) 生物基材料与绿色造纸国家重点实验室 济南 250353) \\ ( ${ }^{b}$ 山东潍坊润丰化工股份有限公司 山东潍坊 261000)
}

\begin{abstract}
摘要 单氟烯烃结构单元广泛存在于药物及天然产物等复杂功能分子中, 同时也是重要的有机合成子, 在医药、生物 和材料等领域具有广泛的应用. 因此, 开发绿色、经济且高效的合成单氟烯烃化合物的方法具有重要的科学意义和现 实价值. 偕二氟烯烃 $\mathrm{C}-\mathrm{F}$ 键官能化是制备单氟烯烃化合物的有效手段, 总结了在可见光氧化还原催化、可见光氧化还 原/过渡金属协同催化条件下实现偕二氟烯烃 $\mathrm{C}-\mathrm{F}$ 键官能化反应的研究进展，主要介绍了底物适用范围、反应机理和 合成应用, 并对它的发展前景进行了展望.
\end{abstract}

关键词＼cjkstart可见光；单氟烯烃; 偕二氟烯烃; C-F 键官能化

\section{Progress in Visible-Light Catalyzed C-F Bond Functionalization of gem-Difluoroalkenes}

\author{
Li, Zhiqing ${ }^{b} \quad$ Qiu, Xiaoyang ${ }^{b} \quad$ Lou, Jiang*,a,b Wang, Qiang*,a \\ ( ${ }^{a}$ State Key Laboratory of Biobased Material and Green Papermaking, Qilu University of Technology \\ (Shandong Academy of Sciences), Jinan 250353) \\ ( ${ }^{b}$ Shandong Weifang Rainbow Chemical Co. Ltd., Weifang, Shandong 261000)
}

\begin{abstract}
Monofluoroalkene structural units widely exist in complex functional molecules such as drugs and natural products. They are also critical organic synthons with broad applications in medicine, biology, materials and other fields. Therefore, it is of great scientific significance and practical value to develop green, economical and efficient methods for synthesizing monofluoroalkenes. Functionalization of the $\mathrm{C}-\mathrm{F}$ bond of gem-difluoroalkenes is an effective method to prepare monofluoroalkenes. The research progress in $\mathrm{C}-\mathrm{F}$ bond functionalization of gem-difluoroalkenes under visible-light redox catalysis and visible-light redox/transition-metal synergistic catalysis, including the substrate scope, reaction mechanism, and synthesis applications, is summarized. Finally, the prospects of this reaction are also discussed.

Keywords visible-light; monofluoroalkene; gem-difluoroalkene; $\mathrm{C}-\mathrm{F}$ bond functionalization
\end{abstract}

氟元素是自然界中电负性最强的元素，向有机分子 的特定位置引入氟原子可以改变母体分子的酸度、细胞 膜通透性、亲脂/亲水平衡和代谢稳定性等性质，对药物 分子在生物体内的吸收、分布及代谢等造成影响, 因此 也逐步成为新型药物设计的一种常用手段 ${ }^{[1]}$. 目前, 市 场上大约 $20 \% \sim 25 \%$ 的药物分子和 $30 \% \sim 40 \%$ 的农药分 子中含有氟原子 [2]. 另外, 含氟化合物在材料科学和生 命科学等领域也有着广泛的应用 ${ }^{[3]}$. 其中, 单氟烯烃是 一类特殊的含氟结构单元, 广泛存在于医药、农药、材
料及精细化学品等功能分子中(图 1) $)^{[4]}$. 在药物化学领 域, 含单氟烯烃结构的药物分子常表现出抗菌、抗肿瘤 或抗炎等药物活性 ${ }^{[4-5]}$; 在生物化学领域, 含单氟烯烃 结构的生物分子可以用作多种酶和活性因子的抑制剂 及生物体内钙膦等矿物质平衡的调节剂, 其与肽键有类 似的空间和电子性质，可以形成氢键，被认为是理想 的、不会水解的肽键的替代物，可以增强肽酶的稳定性， 其刚性结构又能稳定肽分子 ${ }^{[4,6] ;}$ 在农业生产领域，单氟 烯烃化合物可用作各类除草剂和特效农药 ${ }^{[4,7]}$; 在材料

* Corresponding authors. E-mail: jlou@qlu.edu.cn, wangqiang83@qlu.edu.cn

Received June 6, 2021; revised July 1, 2021; published July 19, 2021.

Project supported by the National Natural Science Foundation of China (No. 22001140), the Natural Science Foundation of Shandong Province (No. ZR2020QB002) and the Outstanding Youth Innovation Team Project of Shandong Provincial University (No. 242007040109).

国家自然科学基金(No. 22001140)、山东省自然科学基金(No. ZR2020QB002)和山东省高等学校优秀青年创新团队支持计划(No. 242007040109)资助项 目. 
科学领域, 单氟烯烃化合物可用作新型发光材料和光电 材料 ${ }^{[4,8]}$. 除此之外，单氟烯烃化合物也被广泛应用于有 机合成领域, 是重要的含氟有机合成子, 可以制备各类 链状、碳环及杂环化合物, 为后续相关复杂功能分子的 合成提供便捷、高效的途径 ${ }^{[4,9]}$.

目前, 合成单氟烯烃化合物的方法主要有：(1)基于 醛、酮等羰基化合物的 Julia-Kocienski 烯基化、Peterson 烯基化和 Wittig 反应等; (2)重氮化合物的偶联转化; (3) 卤代烯烃的交叉偶联; (4)烯烃、炔烃及联烯等化合物的 氟化反应. 但这些方法多存在底物需预官能化、反应效 率和选择性低、原子和步骤经济性差等局限性, 极大地 限制了这些方法的进一步发展与应用 ${ }^{[4,10]}$. 近年来, 偕 二氟烯烃化合物因其廉价易得以及反应活性高等特点, 逐渐引起有机合成化学家的研究兴趣, 在过渡金属催化 下经 $\mathrm{C}-\mathrm{F}$ 键活化或 $\beta$-氟消除等方式, 其可以与硅试 剂[11]、喼试剂[12]、Grignard 试剂[13]、有机锌试剂[14]、卤

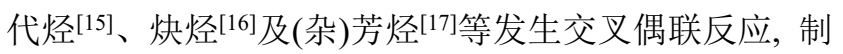
备结构多样的单氟烯烃化合物 ${ }^{[4,18]}$. 然而, 尽管过渡金 属催化偕二氟烯烃 $\mathrm{C}-\mathrm{F}$ 键官能化反应的研究取得了较 大的进展, 但开发更加绿色、高效且经济的单氟烯烃化 合物的合成方法, 仍具有重要的科学意义和现实价值.

在绿色化学理念的推动下, 可见光因其储量丰富以 及清洁安全等优点被广泛应用于有机合成领域, 有力地 推动了可见光化学在天然产物和药物分子等复杂功能 分子合成中的应用 ${ }^{[19]}$. 可见光反应体系一般具有条件
温和、反应效率高、官能团容忍性好及底物普适性广等 优点. 鉴于单氟烯烃化合物的重要性及偕二氟烯烃在制 备单氟烯烃中的广泛应用，本文综述了近年来基于自由 基交叉偶联的可见光氧化还原催化、可见光氧化还原/ 过渡金属协同催化的偕二氟烯烃 $\mathrm{C}-\mathrm{F}$ 键官能化制备结 构多样的单氟烯烃化合物的研究进展.

目前, 可见光催化偕二氟烯烃 $\mathrm{C}-\mathrm{F}$ 键官能化主要 有三条途径(Scheme 1): (1)可见光氧化还原催化剂与偕 二氟烯烃经单电子转移并脱除氟负离子生成高活性的 单氟烯基自由基，进一步与由可见光催化或过渡金属催 化产生的另一自由基物种偶联(Path A); (2)单氟烯基自 由基被过渡金属配合物捕获形成碳-金属键，进而与其

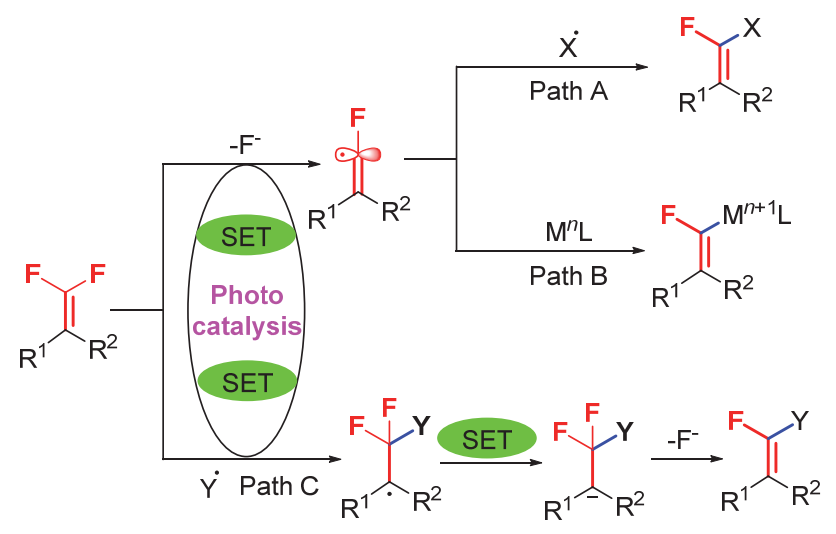

图式 1 可见光催化偕二氟烯烃 $\mathrm{C}-\mathrm{F}$ 键官能化

Scheme 1 Visible-light catalyzed $\mathrm{C}-\mathrm{F}$ bond functionalization of gem-difluoroalkenes

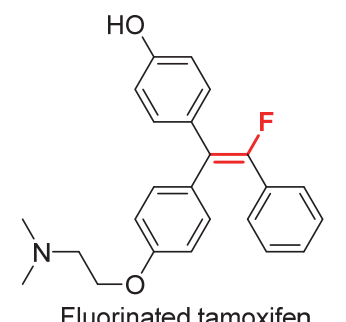<smiles>COc1cc(/C=C(\F)CO)ccc1OC1OCC(CO)(C(O)CO)C(CO)C1(O)CO</smiles>

Fluorinated tamoxifen<smiles>Nc1ccn(C2O[C@H](CO)[C@@H](O)/C2=C/F)c(=O)c1</smiles>

Ribonucleotide reductase inhibitor

Inhibitor of lignification

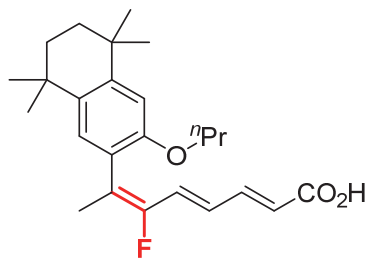

Retinoid $\mathrm{X}$ receptor regulator

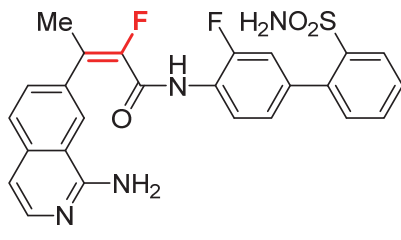

Active factor Xa inhibitor<smiles>CN1CCN(c2c(F)cc3c(=O)c(C(=O)O)cn(/C=C/F)c3c2F)CC1</smiles><smiles>[R]C(c1nc([Al])no1)C(N)C(F)=C1CCCC1</smiles>

Dipeptidyl peptidase IV inhibitor
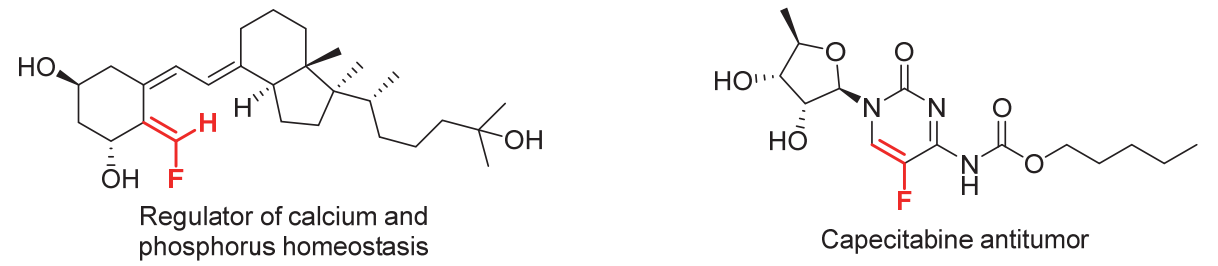

图 1 含单氟烯烃结构单元的代表性生物活性分子

Figure 1 Representative bioactive molecules containing monofluoroalkene structural units 
他亲电试剂偶联(Path B); (3)可见光催化产生活性自由 基物种对偕二氟烯烃选择性加成, 经单电子转移及 $\beta-\mathrm{F}$ 消除实现 $\mathrm{C}-\mathrm{F}$ 键的官能化反应(Path $\mathrm{C}$ ).

\section{1 自由基/自由基交叉偶联反应}

基于自由基活性高及活化能低等特点，过渡金属或 可见光催化的自由基/自由基交叉偶联反应已成为构建碳 一碳键及碳-杂原子键的一种有效途径 ${ }^{[20]}$, 尤其是可见光 催化的 $\mathrm{C}-\mathrm{H}$ 键直接官能化已被广泛应用于有机合成、 高分子材料及生物化学等领域, 底物无需预官能化, 具 有很高的原子和步骤经济性, 符合绿色化学发展理念 ${ }^{[21]}$.

2016 年, Hashmi 等 ${ }^{[22]}$ 以铱配合物 $\operatorname{Ir}\left[\mathrm{dF}\left(\mathrm{CF}_{3}\right) \mathrm{ppy}\right]_{2-}$ (dtbbpy) $\mathrm{PF}_{6}$ 为可见光氧化还原催化剂, 碳酸钠为碱, 在 蓝光照射下, 首次实现了可见光氧化还原催化偕二氟烯 烃 $\mathrm{C}-\mathrm{F}$ 键与三级胺化合物 $\alpha-\mathrm{C}\left(\mathrm{sp}^{3}\right)-\mathrm{H}$ 键的自由基/自 由基交叉偶联反应，为温和条件下制备 $\alpha$-胺甲基官能化 的多取代单氟烯烃化合物 3 提供了一条高效便捷的途径 (Scheme 2). 各种取代的环状或非环状三级胺、三取代 或四取代偕二氟烯烃都能顺利地发生反应, 并且取代基

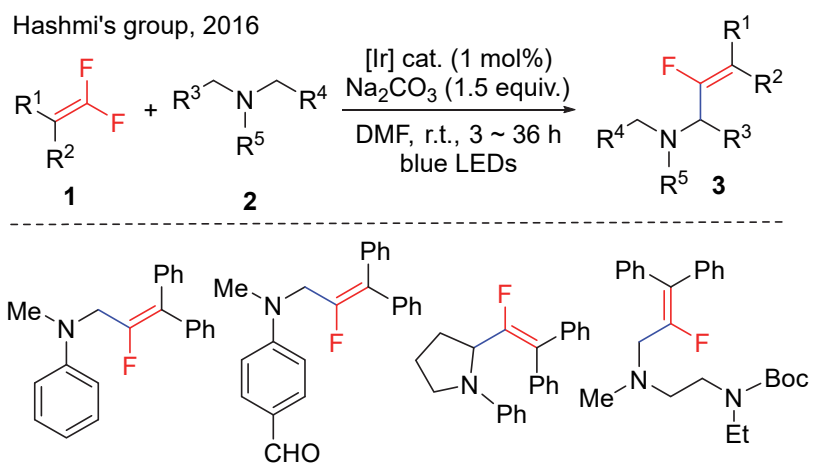

3a, $94 \%(16 h) \quad 3 b, 94 \%(24 h) \quad 3 c, 71 \%(24 h) \quad 3 d, 88 \%(12 h)$

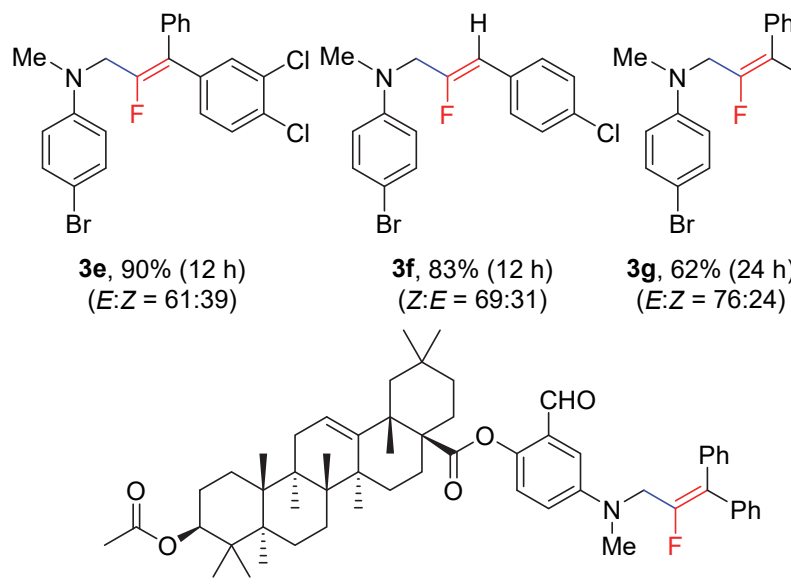

3h, $84 \%$ (1.2 equiv. tertiary amine), from oleanic acid

图式 2 可见光氧化还原催化 $\mathrm{C}\left(\mathrm{sp}^{2}\right)-\mathrm{F}$ 键 $\alpha$-胺甲基化

Scheme 2 Visible-light photoredox catalyzed $\alpha$-aminomethylation of $\mathrm{C}\left(\mathrm{sp}^{2}\right)-\mathrm{F}$ bond
的电性和空间位阻对产物的收率影响不大. 其中，不对 称的四取代偕二氟烯烃底物的反应产物以 $E$ 式为主, 三 取代偕二氟烯烃底物的反应产物以 $Z$ 式为主. 另外, 由 地尔硫卓、西酞普兰、罗格列酮、文拉法辛等药物分子 及双氢青蒿素、齐墩果酸、雄酮等天然产物衍生的三级 胺底物也可以以中等至良好的收率获得目标产物。然 而，苯甲酰甲酸、烷基羧酸或硼酸及三氟硼酸烷基酯等 底物在标准条件下反应时，仅能得到少量的目标产物， 表明短寿命的酰基或烷基自由基不利于后续的自由基 重组过程 ${ }^{[23]}$.

标准条件下向反应体系中加入 2,2,6,6-四甲基哌啶 氧化物(TEMPO)或 1,4-二硝基苯等自由基抑制试剂，反 应被完全抑制，没有目标产物生成，表明反应可能经历 了自由基过程(Scheme 3,a); 向反应体系中加入迈尔克 受体 3-丁烯-2-酮, 可以得到芳基胺与烯酮的偶联产物 4, 表明反应过程中可能生成了 $\alpha$-胺甲基自由基(Scheme 3, $\mathrm{b})$; 以偕二氟烷烃 5 和 7 为底物，没有发生 $\beta-\mathrm{F}$ 消除得到 单氟烯烃化合物，表明反应经历自由基加成/氟消除过 程的可能性很小 (Scheme 3, c, d).

(a)<smiles>CN(C)c1ccc(Br)cc1</smiles><smiles>FC(F)=C(c1ccccc1)c1ccccc1</smiles>
conditions Additive<smiles>CN(C/C(F)=C(\c1ccccc1)c1ccc(Br)cc1)c1ccccc1</smiles>

1a Yield $/ \%$ TEMPO (2 equiv.) 0 1,4-Dinitrobenzene ( 2 equiv.)<smiles>CN(C)c1ccc(Br)cc1</smiles>
2a<smiles>CN(C)c1ccc(Br)cc1</smiles>

2a<smiles>CN(C)c1ccc(Br)cc1</smiles>

$2 \mathrm{a}$

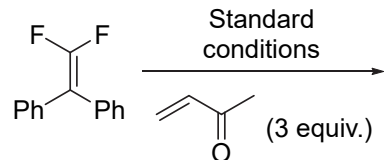

1a

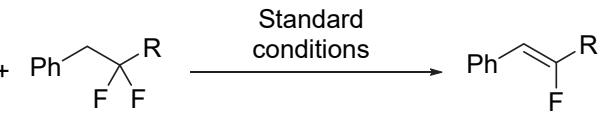

6a, $\mathrm{R}=\mathrm{PO}(\mathrm{OEt})_{2}, 0 \%$ 6b, $\mathrm{R}=\mathrm{COPh}, \quad 0 \%$
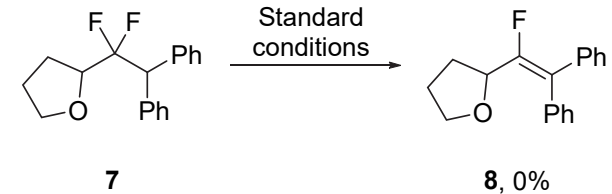

图式 3 光氧化还原催化偕二氟烯烃 $\alpha$-胺甲基化机理研究 Scheme 3 Mechanistic studies of photoredox catalyzed $\alpha$ aminomethylation of gem-difluoroalkenes

基于以上机理研究实验，作者提出了一种可能的反 
应机理(Scheme 4): 在可见光照射下, 光敏剂由基态跃 迁至激发态, 氧化三级胺为自由基阳离子 $\mathbf{A}$, 再脱除一 个质子得到 $\alpha$-胺甲基自由基 $\mathbf{B}$; 偕二氟烯烃被 $\operatorname{Ir}(\mathrm{II})$ 物种 还原为自由基负离子 $\mathbf{C}$, 并脱除氟负离子得到单氟烯基 自由基 $\mathbf{D}$; 最后, $\alpha$-胺甲基自由基 $\mathbf{B}$ 与单氟烯基自由基 $\mathbf{D}$ 经自由基/自由基交叉偶联得到目标产物(Path A), 通过 控制实验排除了 $\alpha$-胺甲基自由基对偕二氟烯烃直接加 成并脱除氟离子的可能性(Path B).

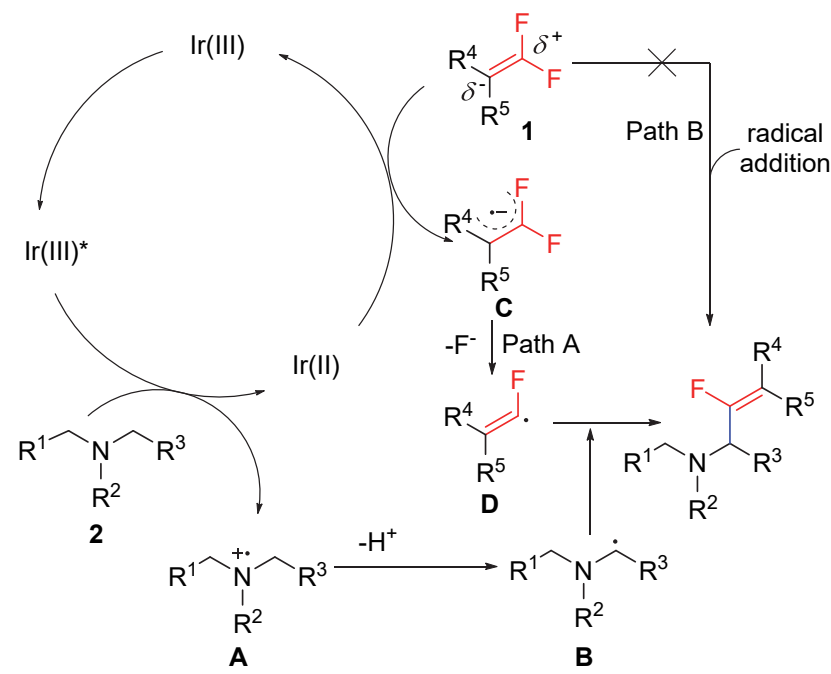

图式 4 偕二氟烯烃 $\alpha$-胺甲基化的反应机理

Scheme 4 Reaction mechanism of $\alpha$-aminomethylation of gemdifluoroalkenes

2019 年, 汪清民等 ${ }^{[24]}$ 报道了可见光氧化还原催化 剂和氢转移催化剂共催化的偕二氟烯烃 $\mathrm{C}-\mathrm{F}$ 键与杂原 子烷烃 9 的 $\alpha$ 位 $\mathrm{C}-\mathrm{H}$ 键的自由基/自由基交叉偶联反应, 以 $\operatorname{Ir}\left[\mathrm{dF}\left(\mathrm{CF}_{3}\right) \mathrm{ppy}\right]_{2}(\mathrm{dtbbpy}) \mathrm{PF}_{6}$ 为可见光氧化还原催化剂, 奎宁 10 为氢转移催化剂, 乙腈和水为溶剂, 在蓝光照射 下，制备了多官能化的单氟烯烃化合物 11, 并将反应底 物由胺类拓展到了含硫、氧等杂原子的化合物(Scheme 5). 条件探索表明, 溶剂中的水可以增加碱 $\mathrm{K}_{3} \mathrm{PO}_{4}$ 的溶 解度, 促进反应的进行, 而仅使用乙腈为溶剂会降低产 物的收率. 偕二氟烯烃底物中芳基取代基上的供电子或 吸电子基团对反应产物的收率影响不大. 叔丁氧羰基 (Boc) 保护的氮杂环丁烷、哌啶、氮杂环庚烷及酰胺保护 的烷基胺等底物都能以中等至良好的收率得到目标产 物 11d 11g. Boc 基团保护的非环状烷基胺底物的反应 性相对较差, 而增加胺类底物用量至 30 equiv. 时, 可以 以较好的收率得到单氟烯烃产物 $11 \mathrm{~h} \sim 11 \mathrm{i}$. 当环状烷基 胺底物含有多个反应位点时, 由于位阻的原因只有一级 碳位点参与反应, 表现出良好的区域选择性. 除胺类底 物外, 含氧、硫等杂原子的烷烃及不含杂原子的均三甲 苯、对二甲苯等底物也可以顺利地得到烷基化单氟烯烃
产物 8 和 $11 \mathrm{j} \sim 11 \mathrm{k}$, 但收率偏低. 另外, 利用发展的方 法还能对娃儿藤碱和利奈唑胺等生物活性分子进行后 期官能化, 为药物制剂的后期单氟烯基化提供了一种可 靠的手段.
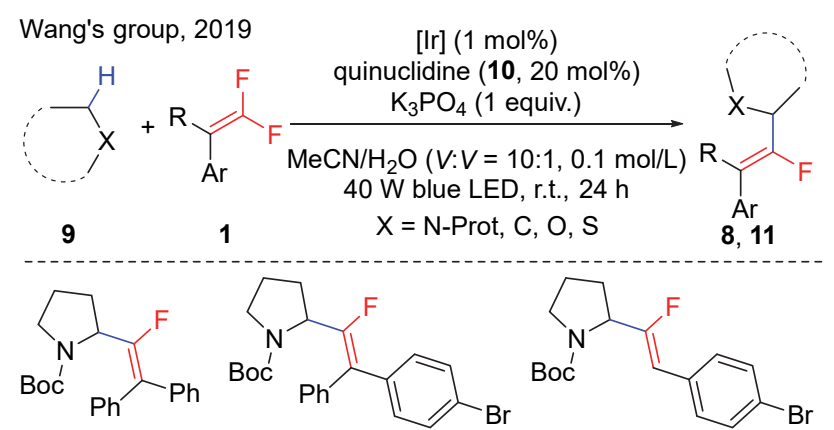

11a, $75 \%$
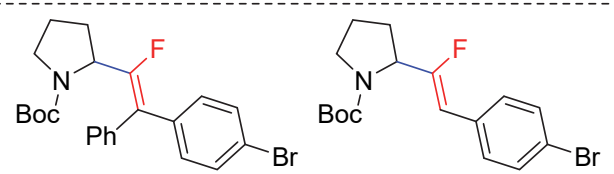

11b, $67 \%, E: Z=2.3: 1$

11c, $77 \%, E: Z=1.1: 1$
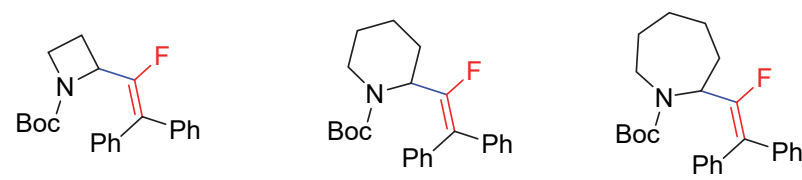

$11 d, 51 \%$<smiles>CN(C)C(=O)N(C)CC(F)=C(c1ccccc1)c1ccccc1</smiles>

$11 e, 39 \%$

11f, $66 \%$
$11 g, 82 \%$

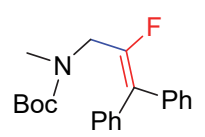

$11 \mathrm{~h}, 76 \%$ (amine, 30 equiv.)<smiles>FC(=C(c1ccccc1)c1ccccc1)C1CCCS1</smiles>

$11 \mathrm{j}, 56 \%$

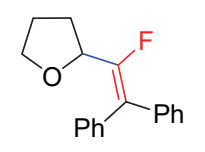

$8,95 \%$

THF as solvent

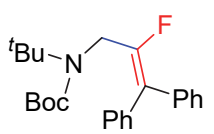

11i, $71 \%$ (amine, 30 equiv.)<smiles>Cc1cc(C)cc(CC(F)=C(c2ccccc2)c2ccccc2)c1</smiles>

$11 k, 31 \%$
图式 5 可见光催化杂原子烷烃 $\alpha$ 位 $\mathrm{C}-\mathrm{H}$ 键的单氟烯基化 Scheme 5 Photoredox-catalyzed $\alpha$-monofluoroalkenylation of heteroatomic alkanes

与 Hashmi 等的报道类似的是, TEMPO 抑制了反应 的进行; 偕二氟乙烯基吲哚底物 12 在标准条件下反应 时, 没有发生 $\mathrm{C}-\mathrm{F}$ 键烷基化，而是得到了分子内环化 产物 13, 表明反应过程中可能产生了单氟烯基自由基 (Eq. 1); 苂光淬灭实验说明奎宁可以淬灭激发态光敏剂, 由此推测反应可能是由奎宁对激发态光敏剂的还原引 发的.

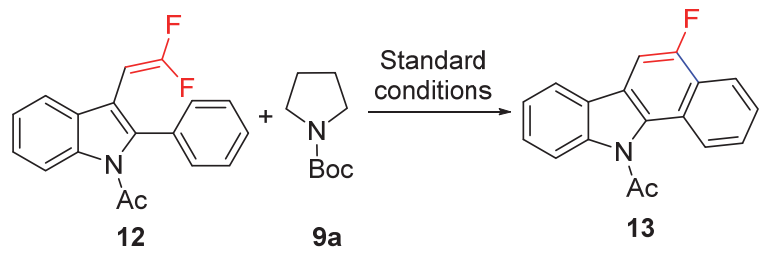

作者提出了可能的反应机理(Scheme 6): 一方面, 激发态光敏剂 $\operatorname{Ir} *(\mathrm{III})\left(E_{1 / 2}[* \operatorname{Ir}(\mathrm{III}) / \operatorname{Ir}(\mathrm{II})]=+1.21 \mathrm{~V}\right.$ vs 


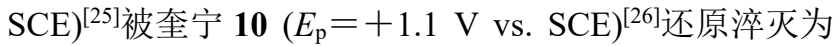
$\mathrm{Ir}(\mathrm{II})$ 物种，奎宁自身被氧化为自由基阳离子 $10 \mathrm{~A}$ ，随后 杂原子烷烃与该自由基阳离子经氢原子转移过程生成 烷基自由基 $\mathbf{A}$; 另一方面, 偕二氟烯烃 $\left(E_{1 / 2}{ }^{\mathrm{red}}=-1.04 \mathrm{~V}\right.$

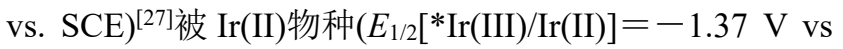

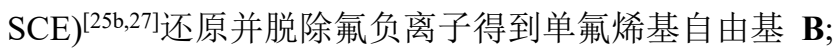
最后，烯基自由基与烷基自由基偶联得到最终产物.

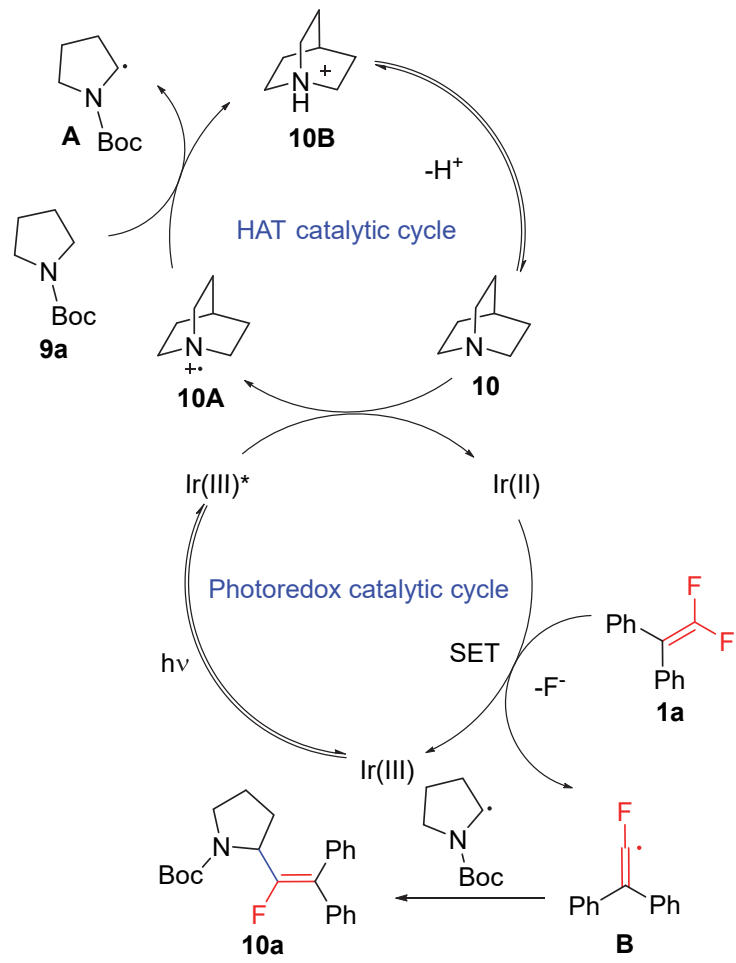

图式 6 杂原子烷烃 $\alpha$-单氟烯基化的反应机理

Scheme 6 Reaction mechanism of $\alpha$-monofluoroalkenylation of heteroatomic alkanes

近年来, 氨基酸等烷基羧酸因其廉价、易得及稳定 等特点, 逐渐发展成为一类新型的烷基自由基前体 ${ }^{[28]}$. 在过渡金属或可见光催化下, 经单电子转移过程, 烷基 羧酸可以脱除一分子二氧化碳得到相应的烷基自由基, 进而对芳烃、烯烃和炔烃加成或与其他自由基偶联等来 构筑高附加值化合物. 特别是可见光催化的烷基羧酸脱 羧偶联反应因其能耗低、污染小、原子和步骤经济性高 等特点而广受关注 ${ }^{[28 b-28 g]}$.

2017 年, 付华等 ${ }^{[29]}$ 报道了可见光氧化还原催化 $\alpha-$ 氨基酸与偕二氟烯烃的脱羧自由基偶联反应, 同样以 $\operatorname{Ir}\left[\mathrm{dF}\left(\mathrm{CF}_{3}\right) \mathrm{ppy}\right]_{2}(\mathrm{dtbbpy}) \mathrm{PF}_{6}$ 为光催化剂, 碳酸锂为碱, 二甲基亚砜(DMSO)为溶剂, 于室温下反应, 合成了各 种类型的 $\alpha$-胺甲基官能化的多取代单氟烯烃化合物 $\mathbf{1 0}$ 和 15 , 反应可以兼容卤素、羟基、砜基、硫醚、酰胺及 磺酰胺等活性基团(Scheme 7). N 原子被保护的脯氨酸、 哌啶酸、甘氨酸、丙氨酸、苯丙氨酸、丝氨酸和甲硫氨
酸等氨基酸都能很好地适应于该反应. 对于偕二氟烯烃 底物而言, 产物的立体选择性与偕二氟烯烃底物的取代 基有较大关系, 不对称的四取代烯烃底物的产物以 $E$ 式 为主，三取代的烯烃底物的产物以 $Z$ 式为主，但 4-碘苯 基和 4-乙氧羰基苯基取代的偕二氟烯烃的反应产物以 $E$ 式为主. 令人遗憾的是, 由二烷基酮和脂肪族醛衍生的 偕二氟烯烃底物在目前的条件下不能发生反应.

Fu's group, 2017
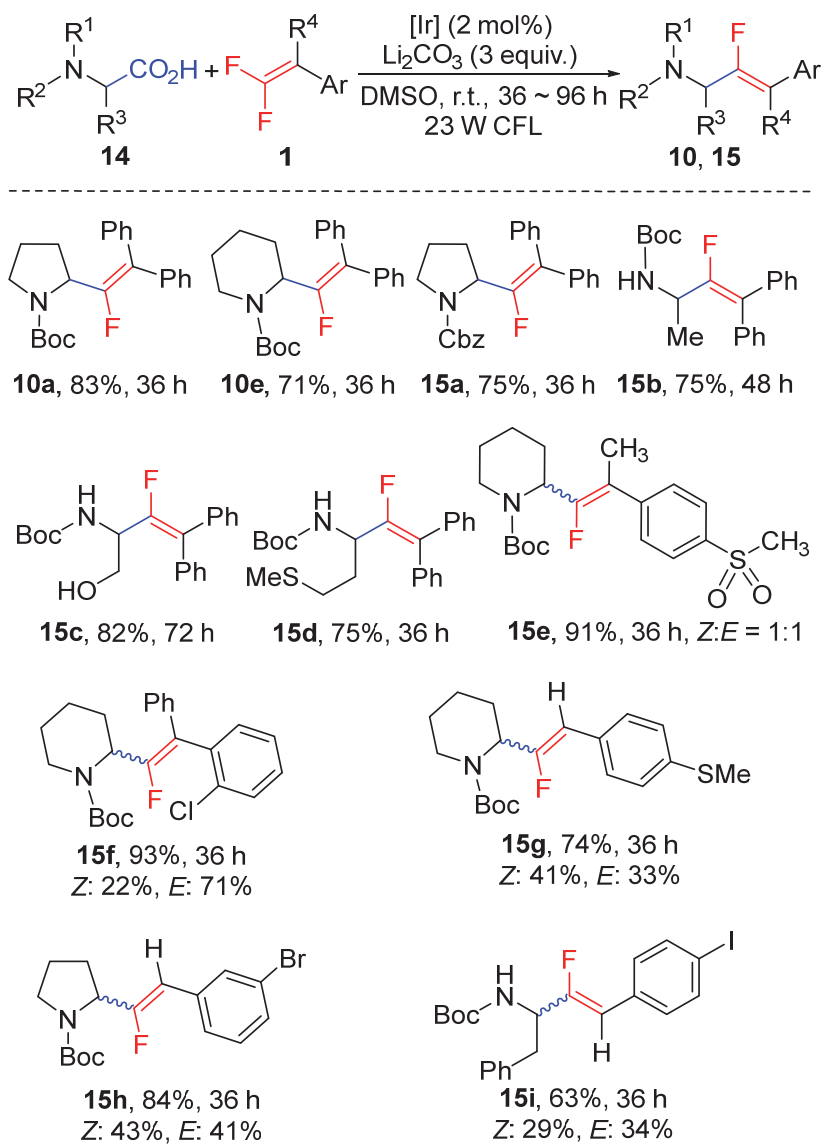

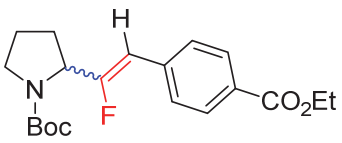

$15 \mathrm{j}, 81 \%, 36 \mathrm{~h}$ Z: $36 \%, E: 45 \%$

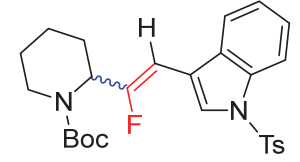

$15 k, 56 \%, 60 \mathrm{~h}$ Z: $29 \%, E: 30 \%$
图式 7 可见光催化 $\alpha$-氨基酸脱羧单氟烯基化 Scheme 7 Visible-light catalyzed decarboxylative monofluoroalkenylation of $\alpha$-amino acids

在碱促进下，具有强氧化性的激发态光敏剂将 $\alpha$-氨 基酸 $\left(E_{1 / 2}{ }^{\mathrm{red}}=+0.95 \mathrm{~V} \text { vs. SCE }\right)^{[25] \text { 氧化并脱除二氧化碳 }}$ 得到烷基自由基 $\mathbf{A}$, 同时得到 $\operatorname{Ir}(\mathrm{II})$ 物种; 在锂离子协助 下，偕二氟烯烃被 $\operatorname{Ir}(\mathrm{II})$ 物种还原并脱除氟负离子得到 单氟烯基自由基 $\mathbf{B}$; 最后，两种自由基交叉偶联得到目 标产物(Scheme 8). 


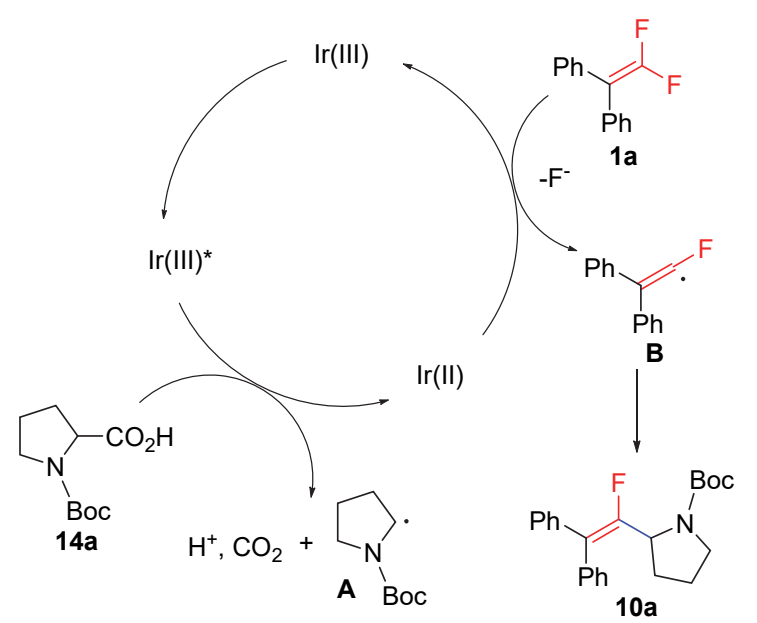

图式 $8 \alpha$-氨基酸脱羧单氟烯基化反应机理

Scheme 8 Reaction mechanism of decarboxylative monofluoroalkenylation of $\alpha$-amino acids

2019 年, 李光明和安光辉等 ${ }^{[30]}$ 以 $2,4,5,6$-四(9-咔唑 基)-间苯二腈(4-CzIPN)为有机光氧化还原催化剂, 碳酸 铯为碱, 实现了偕二氟烯烃 1 与非活化脂肪族羧酸 16 的脱羧偶联反应, 并且反应也可以直接在太阳光照射下 进行(Scheme 9). 偕二氟烯烃中芳基上取代基的位置对 反应结果有较大的影响, 邻位取代的底物比间位、对位

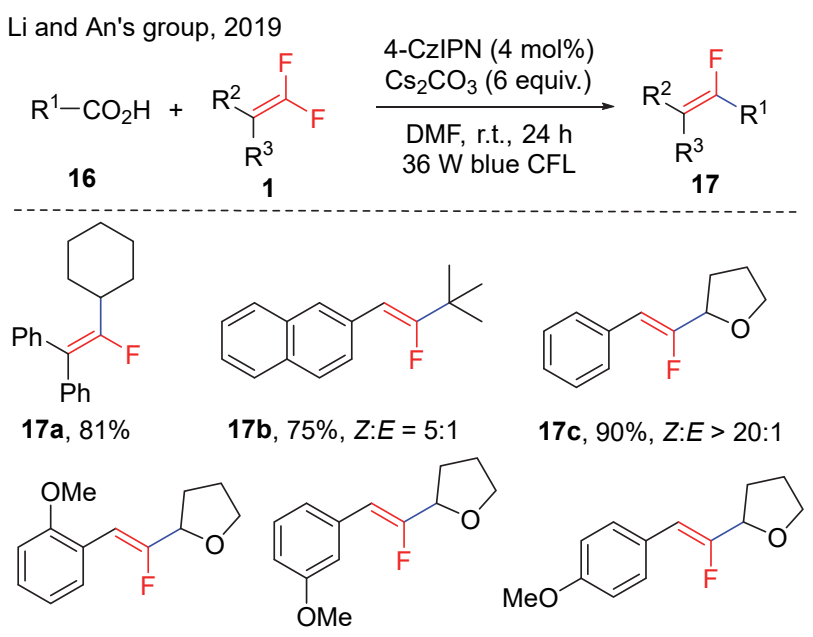

17d, $89 \%, Z: E>20: 117 e, 76 \%, Z: E>20: 1 \quad 17 f, 40 \%, Z: E>20: 1$<smiles>CC(=CC(F)=CC1CCCO1)c1ccccc1</smiles>

$17 \mathrm{~g}, 43 \%, Z: E=1: 1$<smiles>FC(=C(c1ccccc1)C1CCCO1)c1ccccc1</smiles>

17 j, $90 \%$<smiles>FC(CCCc1ccccc1)=C(c1ccccc1)c1ccccc1</smiles>

$17 \mathrm{~h}, 42 \%$

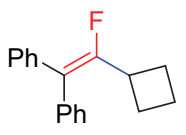

$17 \mathrm{i}, 60 \%$
图式 9 可见光催化偕二氟烯烃和烷基羧酸的脱羧偶联反应 Scheme 9 Visible-light catalyzed decarboxylative cross-coupling of gem-difluoroalkenes with aliphatic carboxylic acids
取代的底物表现出更好的反应活性， $\alpha, \beta$-不饱和偕二氟 烯烃底物也能以中等收率得到单氟二烯产物, 但立体选 择性稍差. 对于羧酸底物而言, 长链一级脂肪酸、碳环 或杂环羧酸、大位阻三级羧酸、苯甲酰甲酸及雷米普利 (ramipril)、吉非罗齐(gemfibrozil)、脱氢枞酸(dehydroabietic acid)、甘草次酸(enoxolone)等生物活性分子都可 以顺利地发生反应得到目标产物. 然而, 脂肪族偕二氟 烯烃和芳香族羧酸底物都不能发生反应.

以环丙基乙酸 16n 为底物进行自由基钟反应，可以 以 $10 \%$ 的收率得到环丙基开环后与偕二氟烯烃的偶联 产物 17n (Scheme 10); 以 TEMPO 为自由基抑制剂, 可 捕获到 2-四氢呋喃甲酸脱羧后形成的烷基自由基; 利用 电子顺磁共振(EPR)技术可以检测到 5,5-二甲基-1-吡咯 啉 $-N$-氧化物(DMPO)与特戊酸产生的叔丁基自由基的 加合物. 这些实验结果表明该反应经历了自由基历程, 也验证了羧酸脱羧可以得到烷基自由基.<smiles>C=CCC/C(F)=C(\c1ccccc1)P(/C=C/CC/C=C/C1CC1COC(=O)CC1CC1)c1ccccc1</smiles>

图式 10 环丙基乙酸参与的自由基钟反应

Scheme 10 Radical clock reaction involving cyclopropylacetic acid

在碱协助下, 激发态光敏剂 $\left[E_{1 / 2}\left(\mathrm{PC}^{*} / \mathrm{PC}^{-}\right)=+1.35\right.$ V vs. SCE $]^{[31]}$ 直接氧化羧酸 $\left(E_{1 / 2}{ }^{\text {red }}=+1.16 \mathrm{~V} \text { vs. SCE }\right)^{[32]}$ 为自由基物种 $\mathbf{A}$, 并脱除一分子二氧化碳得到烷基自由 基 $\mathbf{B}$, 光敏剂自身被还原为负离子; 随后，还原性的负 离子光敏剂 $\left[E_{1 / 2}\left(\mathrm{PC}^{-} / \mathrm{PC}\right)=-1.21 \mathrm{~V} \text { vs. } \mathrm{SCE}\right]^{[31]}$ 将偕二 氟烯烃还原为持久性自由基负离子 $\mathbf{C}$, 并在铯离子的作 用下脱除一个氟负离子得到单氟烯基自由基 $\mathbf{D}$; 最后, 烷基自由基 $\mathbf{B}$ 与单氟烯基自由基 $\mathbf{D}$ 经自由基/自由基交 叉偶联生成烷基官能化的四取代单氟烯烃化合物. 另 外, 烷基自由基 $\mathbf{B}$ 也可能直接和偕二氟烯烃自由基负离 子 $\mathbf{C}$ 偶联, 在持久性自由基效应下脱除氟负离子生成目 标产物(Scheme 11).

除烷基羧酸外，由醛、酮和胺三组分直接缩合制备 的 4-烷基-1,4-二氢吡啶(DHPs, 汉斯酯)也可以作为很好 的烷基化试剂，具有传统烷基化试剂如烷基金属、烷基 卤等不具备的毒性小及官能团容忍性好等优点 ${ }^{[33]}$. 近 几年，随着可见光催化自由基化学的发展，使得该类自 由基参与的多样化反应得以成功实现, 并很好地避免了 高温、强氧化剂及过量醛的使用 [33b-33d].

2020 年, 周明东等 ${ }^{[34]}$ 以 4-烷基-1,4-二氢吡啶 18 为 烷基化试剂, 在可见光催化下实现了偕二氟芳基乙烯的 脱氟烷基化, 以中等至优秀的收率合成了结构多样的多 


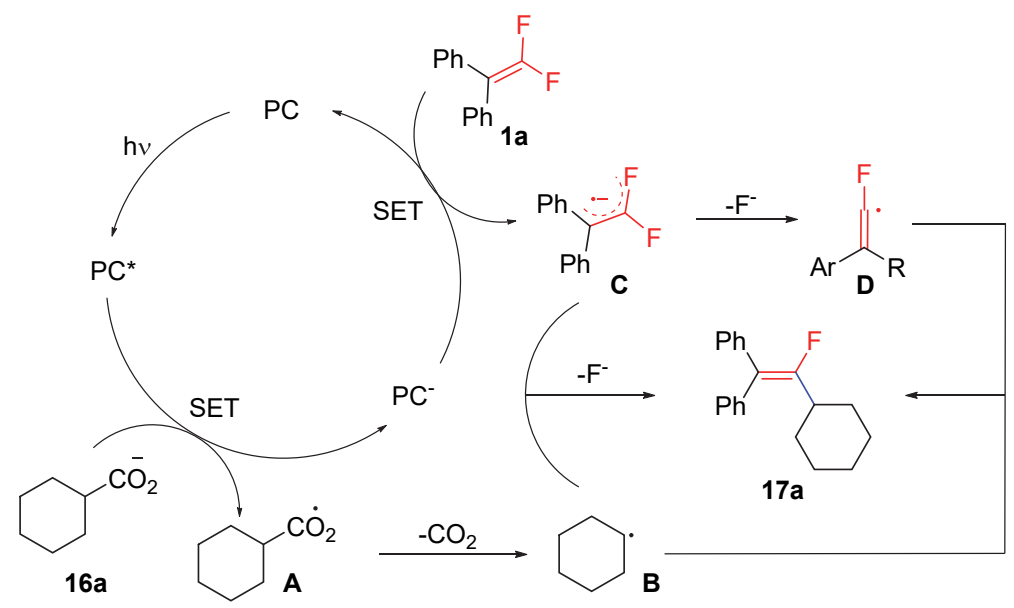

图式 11 自由基脱羧交叉偶联反应机理

Scheme 11 Reaction mechanism of decarboxylative radical cross-coupling

取代烷基化单氟烯烃化合物 19 (Scheme 12). 偕二氟芳 基乙烯底物中芳环上取代基的电子效应和位阻效应对 反应产物的收率没有明显的影响, 一级和二级烷基取代 的二氢吡啶底物都能很好地发生反应. 另外, 由呋喃葡 萄糖或吡喃葡萄糖等糖苷衍生的二氢吡啶底物也适用

Zhou's group, 2020

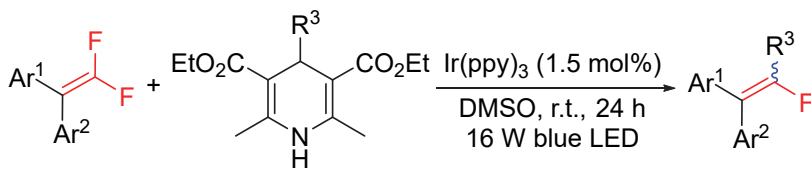
18<smiles>Cc1ccc(C(c2ccc(C)cc2)C(F)C(C)C)cc1</smiles>

$19 a, 85 \%$

$19 b, 88 \%$<smiles>CC(C)/C(=C\c1cccc(Cl)c1)C(C)(F)C(C)(C)C</smiles>

19d, $87 \%, Z: E=1: 1$<smiles>CC(C)/C(F)=C/c1ccc(C(C)(C)C)cc1</smiles>

$19 \mathrm{e}, 50 \%, Z: E=4: 1$

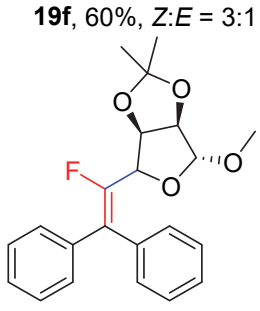

$19 k, 79 \%$

图式 12 可见光催化偕二氟烯烃的脱氟烷基化 Scheme 12 Visible-light catalyzed defluorinative alkylation of gem-difluoroalkenes

于该反应. 当以 4-叔丁基-2,6-二甲基-1,4-二氢吡啶-3,5二甲腈为底物时, 可以实现偕二氟芳基乙烯的脱氟三级 烷基化，但收率偏低.

为了验证反应机理，作者进行了一系列控制实验 (Scheme 13): 以 2 equiv. 1,1-二苯乙烯为自由基捕获剂 时，目标产物的收率仅有 30\%，且可以用 GC-MS 检测 到 1,1-二苯乙烯与烷基自由基的加合产物 20 (Scheme 13，a); 与前述报道类似的是，吲哚基偕二氟烯烃底物 12 只生成了分子内环化产物 13 (Scheme 13, b). 这些结 果表明，反应经历了自由基过程，并且反应过程中产生 了烷基自由基和单氟烯基自由基. 另外，荧光淬灭实验 表明 4-烷基-1,4-二氢吡啶可以淬灭激发态的 $\operatorname{Ir}(\mathrm{ppy})_{3}$, 由此可以推断该反应是由 4-烷基-1,4-二氢吡啶对光催 化剂的还原淬灭引发的.

(a)

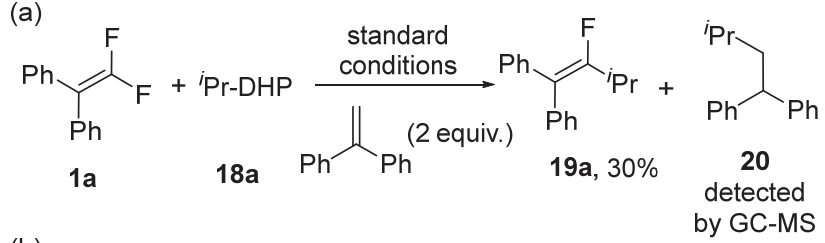

(b)<smiles>Cn1c(-c2ccccc2)c(C=C(F)F)c2ccccc21</smiles>

12 $+{ }^{i} \mathrm{Pr}-\mathrm{DHP}$ standard conditions<smiles>CC(C)(C)Cn1c2ccccc2c2cc(F)c3ccccc3c21</smiles>

$18 a$

图式 13 可见光促进偕二氟烯烃的脱氟烷基化机理研究 Scheme 13 Mechanism studies for visible-light promoted defluorinative alkylation of gem-difluoroalkenes

可能的反应机理如下(Scheme 14): 首先, 激发态光

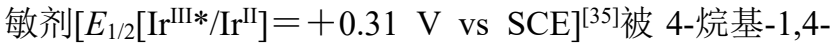

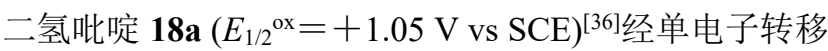


还原淬灭为 $\operatorname{Ir}(\mathrm{II})$ 物种 $\left(E_{1 / 2}\left[\mathrm{Ir}^{\mathrm{III} /} / \mathrm{Ir}^{\mathrm{II}}\right]=-2.19 \mathrm{~V} \text { vs SCE }\right)^{[35]}$, 4-烷基-1,4-二氢吡啶自身经 C-C 键断裂生成烷基自由 基 $\mathbf{A}$; 之后偕二氟烯烃被 $\mathrm{Ir}(\mathrm{II})$ 物种还原为自由基负离子 $\mathbf{C}$, 进一步脱除氟负离子得到单氟烯基自由基 $\mathbf{D}$; 最后, 烷基自由基 $\mathbf{A}$ 与单氟烯基自由基 $\mathbf{D}$ 发生自由基/自由基 偶联生成最终产物 19a.

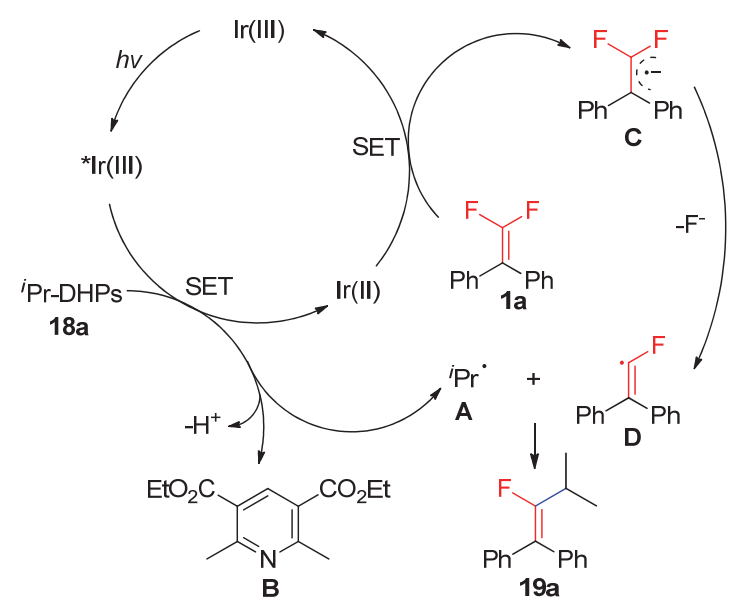

图式 14 可见光促进偕二氟烯烃脱氟烷基化的反应机理 Scheme 14 Possible mechanism for visible-light promoted defluorinative alkylation of gem-difluoroalkenes

基于当今化学领域对复杂功能分子合成效率及成 本的重视, 多组分自由基串联反应因其高效性、原子和 步骤经济性而备受关注, 尤其是烯烃的双官能化已成为 构建含邻位双官能团的复杂烷基化合物的最高效的方 法之一[37].

2019 年, 汪清民课题组 ${ }^{[38]}$ 报道了可见光催化杂化 端烯烃 21、三氟甲亚磺酸钠(22)和偕二氟芳基乙烯 $\mathbf{1}$ 的 三组分自由基串联交叉偶联反应, 实现了杂化烯烃的三 氟甲基和单氟烯基双官能化，合成了一系列三氟甲基烷 基官能化的单氟烯烃化合物 23 (Scheme 15). 反应同样 以 $\operatorname{Ir}\left[\mathrm{dF}\left(\mathrm{CF}_{3}\right) \mathrm{ppy}\right]_{2}(\mathrm{dtbbpy}) \mathrm{PF}_{6}$ 为光敏剂, 反应条件温和, 可以兼容酰胺、酯基及杂环等官能团. 杂化端烯烃上取 代基的类型对反应效率有较大的影响, 烯胺、烯醇醚及 由胆甾醇、维生素 $\mathrm{E}$ 和苯溴马隆等生物活性分子衍生的 端烯烃底物都可以顺利地发生反应, 以中等至良好的收 率得到目标产物, 而其他杂原子官能化的端烯烃, 如烯 基硅醚和烯基硫醚, 以及没有杂原子取代的烯烃, 如苯 乙烯和脂肪族烯烃等, 都不能反应得到目标产物, 这说 明氧、氮等杂原子取代的烯基结构对该反应的顺利进行 具有明显的促进作用. 各种 $\beta, \beta$-二芳基取代的偕二氟烯 烃底物均能较好地反应, 且 $\beta$-芳基- $\beta$-甲基偕二氟烯烃 底物可以得到单一的 $Z$ 式构型产物. 含供电子或吸电子 基的芳基或苯并噻吩、喹啉、吲哚等杂芳基官能化的三 取代偕二氟烯烃与乙烯基苯醚、三氟甲亚磺酸钠反应, 可以以中等收率得到各类三氟甲基和氟原子官能化的
烯丙基化合物.

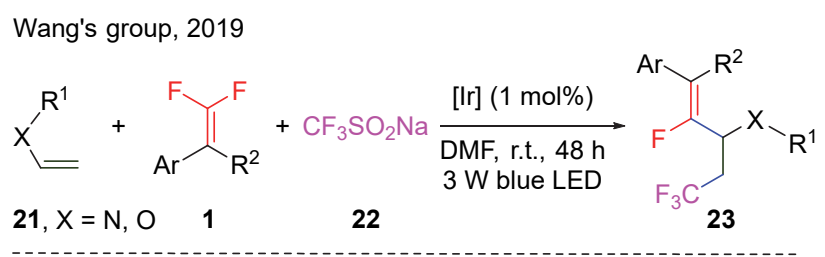

21, $\mathrm{X}=\mathrm{N}, \mathrm{O}$<smiles>O=[N+]([O-])C(CC(F)(F)F)C(=C(c1ccccc1)c1ccccc1)c1ccccc1</smiles>

23b, $50 \%$<smiles>FC(=C(c1ccccc1)C(CC(F)(F)F)Oc1ccccc1)c1ccccc1</smiles>

23c, $85 \%$<smiles>O=C(OC(CC(F)(F)F)c1ccccc1)C(=C(F)c1ccccc1)c1ccccc1</smiles>

23d, $68 \%$

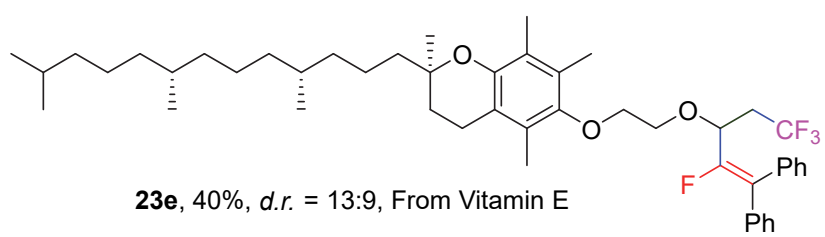<smiles>CC(C(Oc1ccccc1)c1ccc(C(F)(F)F)cc1)C(F)(F)F</smiles>

23f, $60 \%, Z: E=63: 37 \mathbf{2 3 g}, 63 \%, Z: E=63: 37 \quad$ 23h, $49 \%, Z$ only

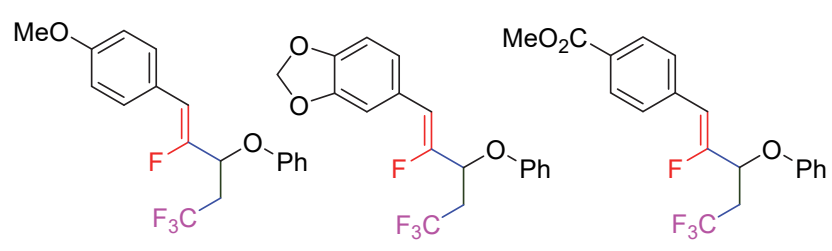

23i, $54 \%, Z$ only

23j, $74 \%, Z: E=68: 32$ 23k, $68 \%, Z: E=55: 45$

图式 15 可见光催化三组分串联反应

Scheme 15 Three-component tandem reactions catalyzed by visible-light

在可见光照射下, 光敏剂 $\operatorname{Ir}(\mathrm{III})$ 由基态被激发为强 氧化性的激发态 $\operatorname{Ir}(\mathrm{III})^{*}$, 与三氟甲亚磺酸钠(22)经单电 子转移过程生成三氟甲基自由基 $\mathbf{A}$ 和 $\operatorname{Ir}(\mathrm{II})$ 物种; 然后, 三氟甲基自由基 $\mathbf{A}$ 对富电子杂化烯烃 $21 \mathrm{c}$ 加成得到杂原 子稳定的烷基自由基 $\mathbf{B}$; 之后, 偕二氟烯烃 1a 被 $\operatorname{Ir}(\mathrm{II})$ 物种还原为自由基负离子 $\mathbf{C}$ 并脱除氟负离子得到单氟 烯基自由基 $\mathbf{D}$; 最后, 烷基自由基 $\mathbf{B}$ 与单氟烯基自由基 D 重组偶联生成目标产物(Scheme 16). 该方法为三氟甲 基和氟原子官能化的结构多样性的烯丙基化合物的合 成提供了有力的基础.

氟原子或含氟基团官能化的有机嗍烷可以参与多 种类型的衍生化反应，是制备高附加值有机氟化物的多 功能合成子 ${ }^{[39]}$. 通过多氟有机化合物的选择性 $\mathrm{C}-\mathrm{F}$ 键 


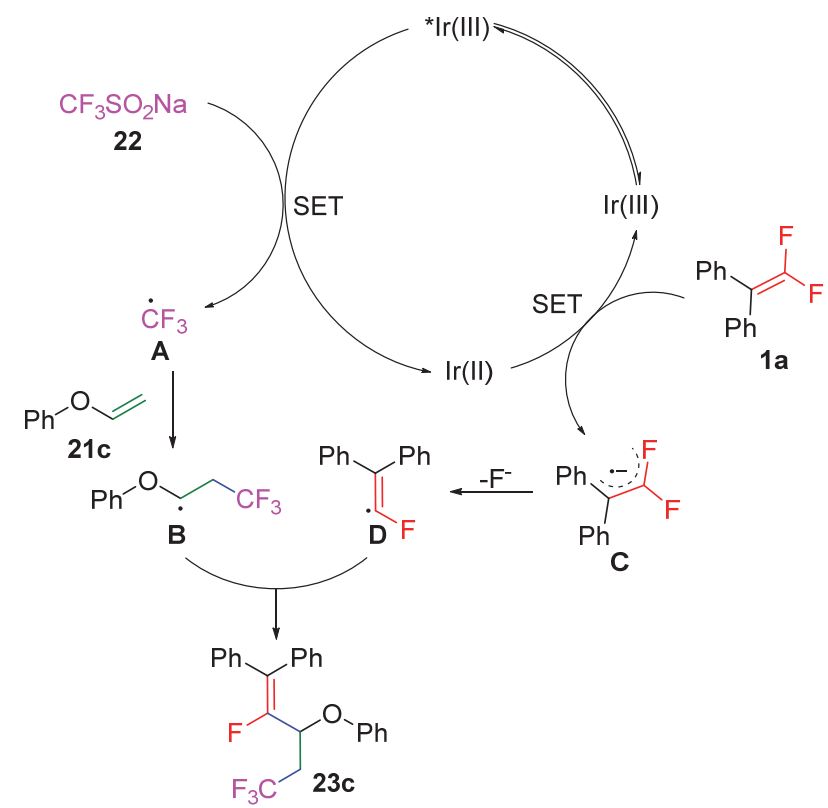

图式 16 可见光催化三组分串联反应机理

Scheme 16 Reaction mechanism of three-component tandem reactions catalyzed by visible-light

嗍化是制备此类化合物最有效的方式之一, 而由于 $\mathrm{C}$ $\mathrm{F}$ 键的惰性、金属一氟中间体的高键能、嗍试剂易亲氟等 因素导致温和条件下脱氟硼化存在极大的挑战性 ${ }^{[40]}$.

2020 年, 吴杰课题组 ${ }^{[41]}$ 报道了可见光诱导偕二氟 烯烃的选择性脱氟硼化反应. 反应以 $\operatorname{Ir}\left[\mathrm{dF}\left(\mathrm{CF}_{3}\right) \mathrm{ppy}\right]_{2}-$ (5,5'-dFbpy) $\mathrm{PF}_{6}$ 为可见光氧化还原催化剂, 2 -颈基丙酸 乙酯(HAT, 24)为氢原子转移试剂、氮杂环卡宾硼烷 25 为硼化试剂, 在蓝光照射下, 通过 $\mathrm{C}-\mathrm{F}$ 键官能化制备 了一系列 $\alpha$-氟烯基硼烷 26 (Scheme 17). 对称或不对称 的偕二氟烯烃都可以顺利地发生反应, 并且可以兼容卤 素、硫醚、酚羟基及胺基等活性官能团. 除此之外, 多 氟芳烃和 $\alpha$-三氟甲基烯烃也适用于该反应，得到相应的 芳基硼烷或烯丙基硼烷化合物。

在蓝光照射下, 一方面, 24 被激发态光催化剂经单 电子转移氧化为亲电性硫自由基 $\mathbf{A}$, 基于硫自由基与氮 杂环卡宾硼烷间的动力学极性匹配效应, 硫自由基瞳取 嗍烷 $\mathbf{2 5}$ 的氢生成嗍自由基 $\mathbf{B}$; 另一方面, 偕二氟烯烃被 光催化剂还原为自由基负离子 $\mathbf{C}$, 与嗍自由基 $\mathbf{B}$ 发生自 由基偶联并脱除氟负离子得到目标产物，或者自由基负 离子 $\mathbf{C}$ 先离去氟负离子再与嗍自由基 $\mathbf{B}$ 偶联. 值得注意 的是，嗍自由基也可能直接对偕二氟烯烃加成，再被光 催化剂还原为碳负离子, 进而脱除氟负离子得到目标产 物(Scheme 18).

\section{2 自由基/过渡金属交叉偶联反应}

随着可见光氧化还原催化在有机合成领域的快速 发展，可见光氧化还原催化与过渡金属协同催化的策略

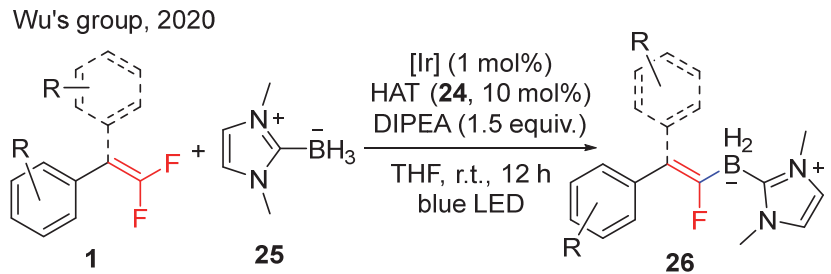

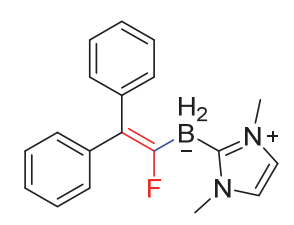

26a, $80 \%$<smiles>Cn1cc[n+](C)c1BC(F)=C(c1ccc(Cl)cc1)c1ccc(Cl)cc1</smiles>

26c, $87 \%$<smiles>Cn1cc[n+](C)c1[B]C(F)=Cc1ccc(O)cc1</smiles>

26e, $67 \%, E: Z=50: 50$<smiles>Cn1cc[n+](C)c1BC(F)=C(c1ccc(F)cc1)c1ccc(F)cc1</smiles>

$26 b, 82 \%$<smiles>Cn1cc[n+](C)c1[B]C(F)=Cc1ccc(S(C)(=O)=O)cc1</smiles>

26d, $75 \%, E: Z=40: 60$<smiles>Cn1cc[n+](C)c1[B]C(F)=Cc1ccc(P)cc1</smiles>

26f, $62 \%, E: Z=40: 60$
图式 17 可见光诱导偕二氟烯烃选择性脱氟硼化反应 Scheme 17 Visible-light-induced selective defluoroborylation of gem-difluoroalkenes

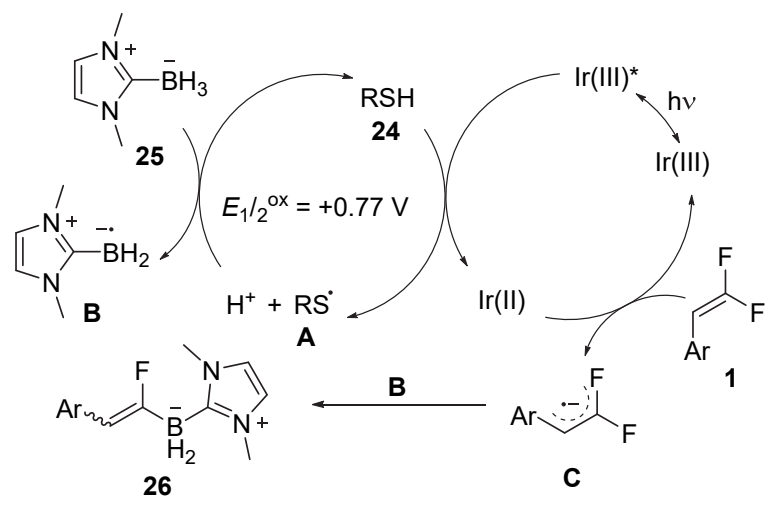

图式 18 选择性脱氟硼化反应机理

Scheme 18 Reaction mechanism of selective defluoroborylation

表现出了单一催化体系不具备的功效，该策略利用可见 光氧化还原催化与过渡金属催化剂在较为温和的条件 下协同作用产生高活性的反应中间体，实现单一催化体 系难以完成的反应, 为碳一碳键及碳一杂原子键的构建提 供了一种新思路 ${ }^{[42]}$.

2019 年，冯超等 ${ }^{[43]}$ 报道了可见光氧化还原/过渡金 属双重催化的偕二氟芳基乙烯 $\mathrm{C}-\mathrm{F}$ 键的羧基化反应. 该反应以 $\operatorname{Ir}\left[\mathrm{dF}\left(\mathrm{CF}_{3}\right) \mathrm{ppy}\right]_{2}(\mathrm{dtbbpy}) \mathrm{PF}_{6}$ 为可见光氧化还原 
催化剂, $\mathrm{PdCl}_{2}$ 为过渡金属催化剂, 三苯基膦为配体, 二 异丙基乙基胺为还原剂, 碳酸铯为碱, 在二氧化碳氛围 中实现了偕二氟芳基乙烯的脱氟羧基化, 制备了多官能 化的 $\alpha$-氟丙烯酸和 2-氟-1,3-丁二烯化合物 27 (Scheme 19). 尽管在可见光催化体系中常用镍催化剂来实现碳-

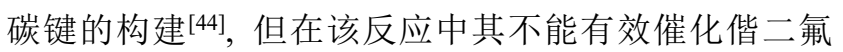
烯烃 $\mathrm{C}-\mathrm{F}$ 键的羧基化. 该反应表现出较为明显的配体 效应, 其他单膦或双膦配体, 如三环己基膦、2-二环己 基磷-2',4',6'-三异丙基联苯 (Xphos)、4,5-双 (二苯基 膦)-9,9-二甲基氧杂葸(Xantphos)等, 仅能以中等收率得 到目标产物. 另外, 由于钯/膦配合物在光激发下自身就 可以作为一种有效的单电子供体来催化反应 ${ }^{[45]}$, 因此 为了验证铱光敏剂存在的必要性, 作者在标准条件下进 行控制实验，结果表明无铱光敏剂时反应不能发生，从 而证明了铱光敏剂的必要性.

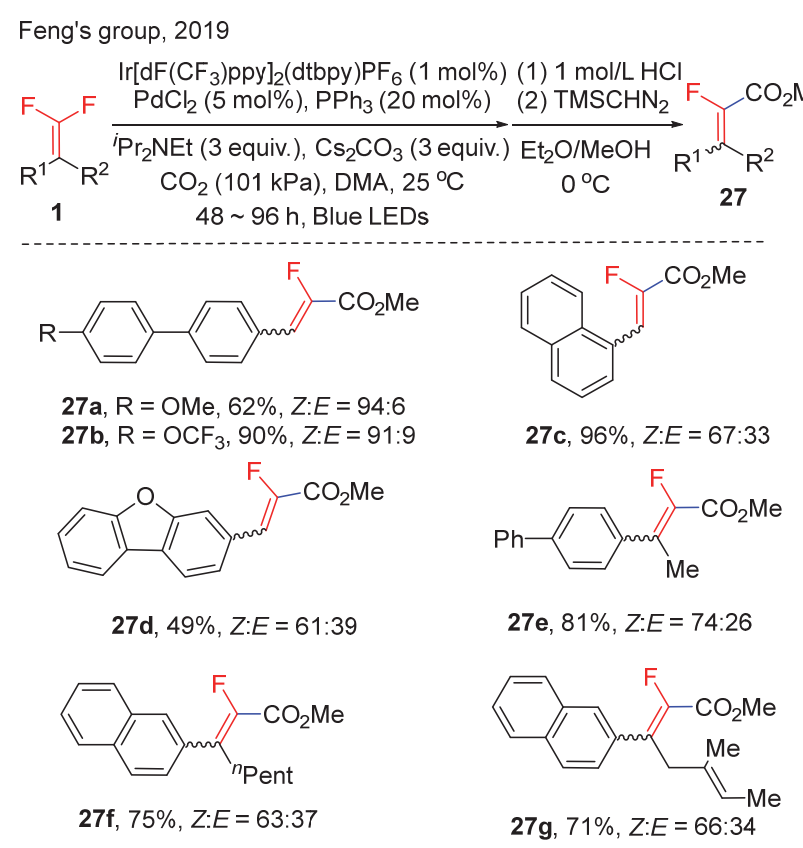

图式 19 可见光/钯协同催化 $\mathrm{C}-\mathrm{F}$ 键羧基化 Scheme 19 Visible-light/palladium co-catalyzed $\mathrm{C}-\mathrm{F}$ bond carboxylation

三取代偕二氟烯烃底物中联苯基团上连有供电子 基如甲氧基时, 反应产物 $27 \mathrm{a}$ 的收率偏低, 可能是由于 此类底物所形成的氟烯基自由基具有较低的还原势能, 在随后的羧基化过程中与钯催化剂或光催化剂的相互 作用变弱, 导致产物收率降低; 而当联苯基团上连有吸 电子基如三氟甲氧基时，反应产物 $27 \mathbf{b}$ 的收率可达 $90 \%$. 当 $\mathrm{R}^{1}$ 取代基为大位阻的 1-䒺基时, 反应仍可以优异的 收率获得最终产物 27c, 但立体选择性变差 $(Z: E=$ $67 ： 33)$. 与三取代偕二氟烯烃底物相比, 四取代的偕 二氟芳基乙烯底物反应需要更长的反应时间, 但可以容
忍长链烷基及烯基等官能团 $(27 \mathrm{e} \sim 27 \mathrm{~g})$. 除二氧化碳外, 其他羰基亲电试剂如芳基酮化合物 28 也可参与反应, 但得到的是脱氟烯基化产物 $\mathbf{3 0}$, 说明在反应过程中发 生了进一步的脱水反应，且有趣的是溴取代基并没有发 生反应, 而是被保留了下来, 说明该反应经历的过程与 Iwasawa 等 [46]提出的芳基溴或氯的羧基化历程不同 (Scheme 20, a). 同样, $\alpha$-氟- $\alpha$-溴烯烃底物 31 在四(三苯 基膦)钯催化下也没有发生 $\mathrm{C}-\mathrm{Br}$ 键的羧基化, 说明反 应中没有发生双电子氧化加成(Scheme 20, b).

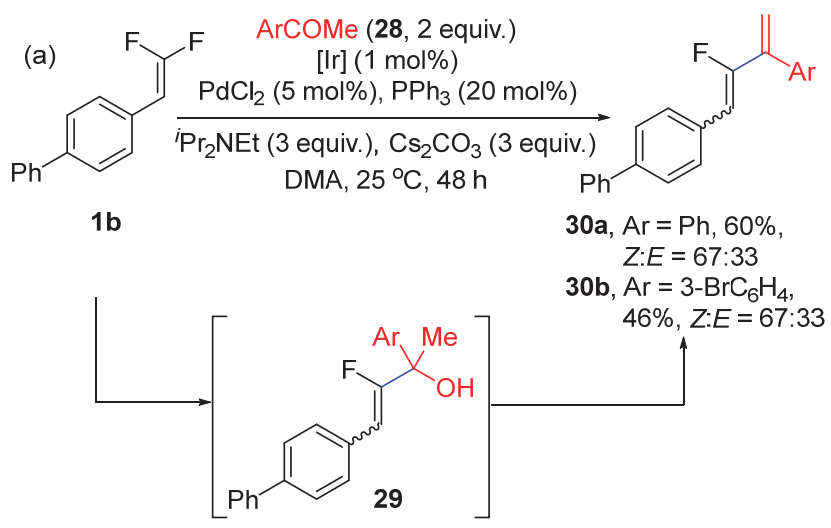

(b)

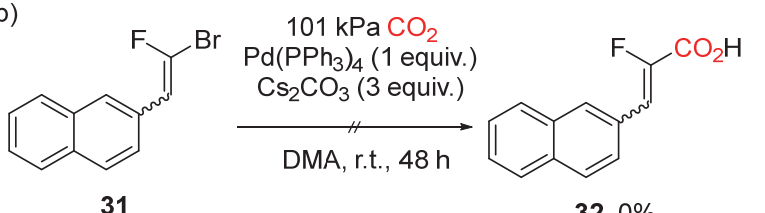

$32,0 \%$

图式 20 芳基酮参与的脱氟羧基化反应

Scheme 20 Defluorocarboxylation of aryl ketones

在对反应机理进行研究时，发现自由基抑制试剂 TEMPO 和 2,6-二叔丁基对甲酚(BHT)均对目标反应的 进行具有较强的抑制作用(Scheme 21, a); 在氮气气氛 下，向反应体系中加入 2 equiv.汉斯酯，反应得到了脱氟 氢化产物 34, 但立体选择性发生了明显的变化, 说明氟 烯基自由基中间体的构型具有易变性，并且这类氟烯基 自由基物种与氟烯基钯中间体相比具有不同的动力学 反应性曲线(Scheme 21, b); 另外, 当向反应体系中加入 3 equiv. $\mathrm{D}_{2} \mathrm{O}$ 时并没有发生 $\mathrm{C}-\mathrm{F}$ 键的氝化, 由此排除了 由氟烯基碳负离子中间体实现羧基化的可能(Scheme $21, \mathrm{c})$.

基于上述机理研究结果, 作者提出了可能的反应机 理(Scheme 22): 激发态光敏剂被 ${ }^{i} \mathrm{Pr}_{2} \mathrm{NEt}$ 还原淬灭为 $\mathrm{PC}^{-1}$, 随后 $\mathrm{PC}^{-1}$ 还原偕二氟烯烃为单氟烯基自由基 $\mathbf{A}$, 并被钯配合物捕获形成烯基钯(I)物种 $\mathbf{B}$; 之后, $\mathrm{CO}_{2}$ 与 该物种配位及迁移插入得到羧基钯( I)物种 $\mathbf{D}$; 最后, $\mathrm{PC}^{-1}$ 与羧基钯(I)物种 $\mathbf{D}$ 经单电子转移生成最终产物及 
(a)<smiles>FC(F)=Cc1ccc(-c2ccccc2)cc1</smiles>

standard conditions radical scavenger TEMPO (2 equiv.) BHT (2 equiv.)

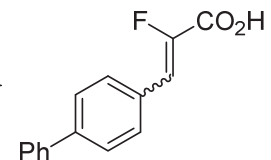

$33 a, 15 \%, Z: E=87: 13$ 33b, 38\%, Z:E $=84: 16$

(b)<smiles>FC(F)=Cc1ccc(-c2ccccc2)cc1</smiles>

Hantzsch ester (2 equiv.), $\mathrm{N}_{2}$

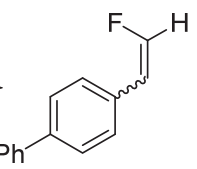

34, $18 \%, Z: E=18: 82$

1b w/o 5 mol\% $\mathrm{PdCl}_{2} / 20$ mol\% $\mathrm{PPh}_{3}$

$34,16 \%, Z: E=25: 75$

(c)

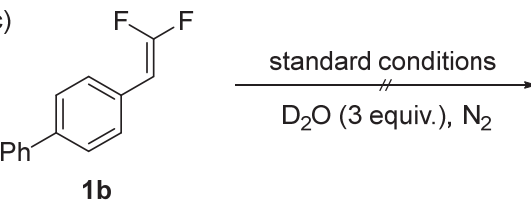

图式 21 芳基酮参与的脱氟羧基化反应机理研究

Scheme 21 Mechanistic studies of defluorocarboxylation of aryl ketones

再生 $\operatorname{Pd}(0) \mathrm{L}$ 完成催化循环. 另外, 作者推测由于 $\operatorname{Pd}(0)$ 与氟烯基自由基的结合存在可逆性导致 $Z$ 式和 $E$ 式烯基 钯中间体可以相互转化, 而动力学上更倾向于形成 $E$ 式 烯基钯，所以最终的产物以 $Z$ 式为主.

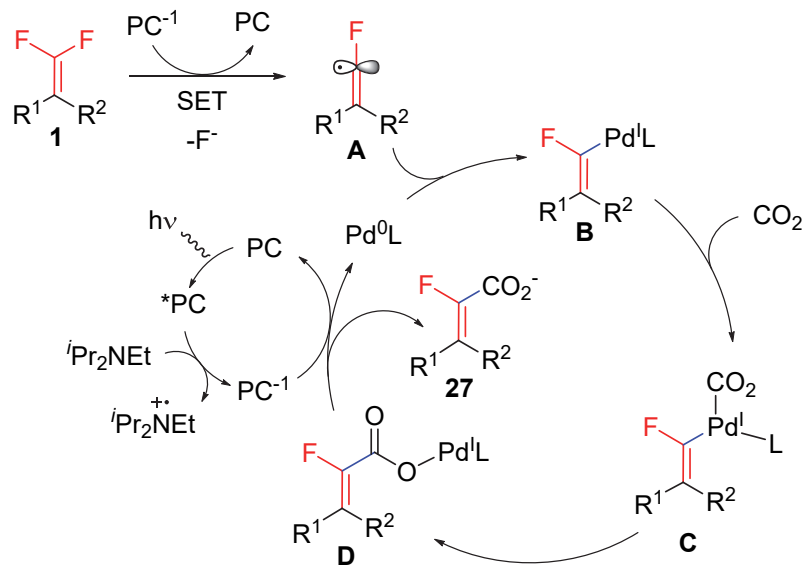

图式 22 芳基酮参与的脱氟羧基化反应机理

Scheme 22 Reaction mechanism of defluorocarboxylation of aryl ketones

\section{3 自由基加成 $/ \beta-F$ 消除反应}

2018 年, Loh 小组 ${ }^{[47]}$ 以 以 $\left[\operatorname{Ir}\left(\mathrm{dF}\left(\mathrm{CF}_{3}\right) \mathrm{ppy}\right)_{2}\left(5,5^{\prime}-\right.\right.$ $\left.\left(\mathrm{CF}_{3}\right)_{2} \mathrm{bpy}\right) \mathrm{PF}_{6}$ 为可见光氧化还原催化剂, 三氟甲亚磺 酸钠(22)为三氟甲基化试剂, 在可见光催化下实现了偕 二氟芳基乙烯的脱氟三氟甲基化，以中等收率制备了 $\alpha$ 氟- $\alpha$-三氟甲基芳基乙烯化合物 35, 立体选择性 $Z / E$ 比高 达 99：1 (Scheme 23). 溶剂的极性对反应影响较大, 小 极性溶剂如丙酮等比大极性溶剂如 $N, N$-二甲基乙酰胺 (DMA)、二甲基亚砜(DMSO)等更利于反应的进行. 反应

对各类取代基均有较好的容忍性，当偕二氟芳基乙烯的 芳基上连有酯基、氭基、磷酸酯基及酰胺基等吸电子取 代基时，反应都能以优异的立体选择性得到目标产物 $(Z ： E=96 ： 4 \sim 99 ： 1)$; 但当芳基上连有供电子取代基 时，反应则不能顺利地发生，可能是由于在反应过程中 产生的 $\alpha$-三氟甲基碳负离子具有较低的活性或稳定性. 该反应所呈现的优异的立体选择性结果与之前理论计 算的报道相一致 ${ }^{[48],} Z$ 式构型具有更高的热力学稳定性 且在反应过程中其过渡态具有更低的反应能垒，并且三 氟甲基较氟原子具有更大的空间位阻，倾向于与芳基基 团处于反式位置.

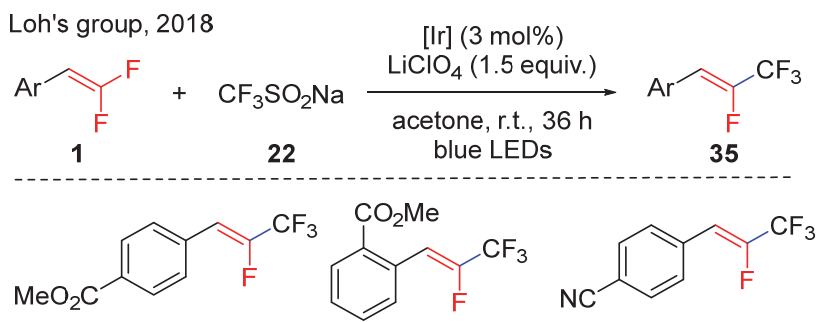

35a, $52 \%, Z: E=99: 1 \quad 35 b, 51 \%, Z: E=96: 4$ 35c, $53 \%, Z: E=95: 5$<smiles>CCNC(=O)c1ccc(/C=C(\F)C(F)(F)F)cc1</smiles>

35d, $47 \%, Z: E=99: 1 \quad 35 \mathrm{e}, 51 \%, Z: E=96: 4$ 35f, $47 \%, Z: E=91: 9$

图式 23 可见光催化偕二氟烯烃 $\mathrm{C}-\mathrm{F}$ 键三氟甲基化 Scheme 23 Visible-light catalyzed trifluoromethylation of C$\mathrm{F}$ bond of gem-difluoroalkenes

在反应中，三氟甲亚磺酸(22)首先被激发态光敏剂 经单电子转移氧化为三氟甲基自由基 $\mathbf{A}$, 光敏剂自身被 还原为 $\operatorname{Ir}(\mathrm{II})$ 物种; 随后，三氟甲基自由基 $\mathbf{A}$ 对偕二氟烯 烃选择性加成得到烷基自由基 $\mathbf{B}$, 该自由基被 $\operatorname{Ir}(\mathrm{II})$ 物种 还原为烷基碳负离子 $\mathbf{C}$, 经 $\beta-\mathrm{F}$ 消除得到目标产物 (Scheme 24).

碳一硫键是一类重要的碳一杂原子化学键，广泛存在 于药物分子、天然产物和有机功能材料中 ${ }^{[49]}$, 碳一硫键 也可以转化为结构多样的官能团 ${ }^{[50]}$, 因此开发有效的 碳一硫键的构建方法具有重要的意义.

2019 年, 夏吾昫等 ${ }^{[51]}$ 在可见光促进下实现了偕二 氟烯烃与硫醇 36 的脱氟交叉偶联反应，制备了含硫基 团官能化的单氟烯烃化合物 37 (Scheme 25). 反应溶剂 对反应的收率和立体选择性有影响较大, 三取代偕二氟 烯烃底物需以甲苯为溶剂, 四取代偕二氟烯烃底物需以 乙腈为溶剂，当以二氯甲烷为溶剂时，两类反应都不能 正常进行．各种类型的芳基、杂芳基偕二氟烯烃和芳基、 烷基硫醇都能很好的适用于该反应，并且由脱氢枞酸及 薯蓣㿝素等天然产物衍生的硫醇底物也可以以中等至 良好的收率得到目标产物. 


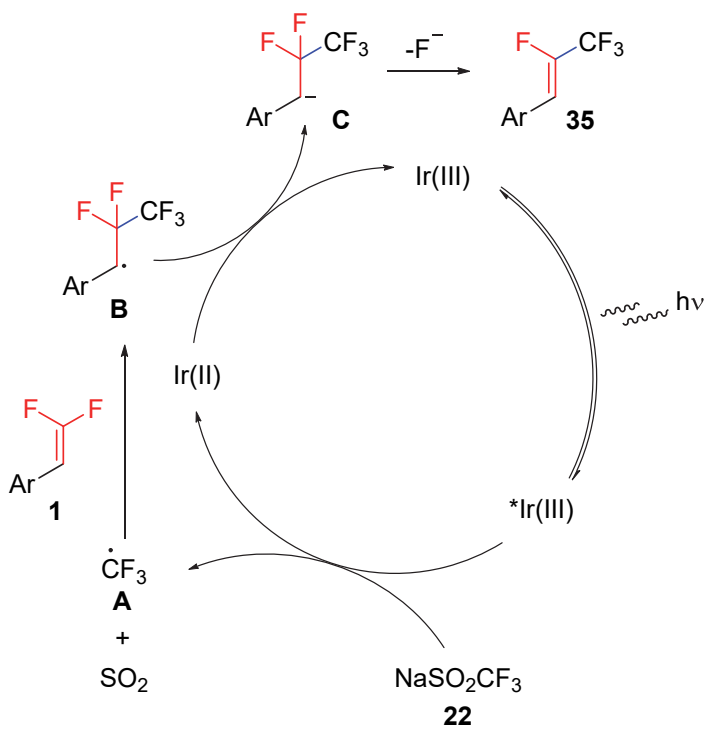

图式 24 偕二氟烯烃 $\mathrm{C}-\mathrm{F}$ 键三氟甲基化反应机理 Scheme 24 Reaction mechanism for trifluoromethylation of $\mathrm{C}-\mathrm{F}$ bond of $\mathrm{gem}$-difluoroalkenes

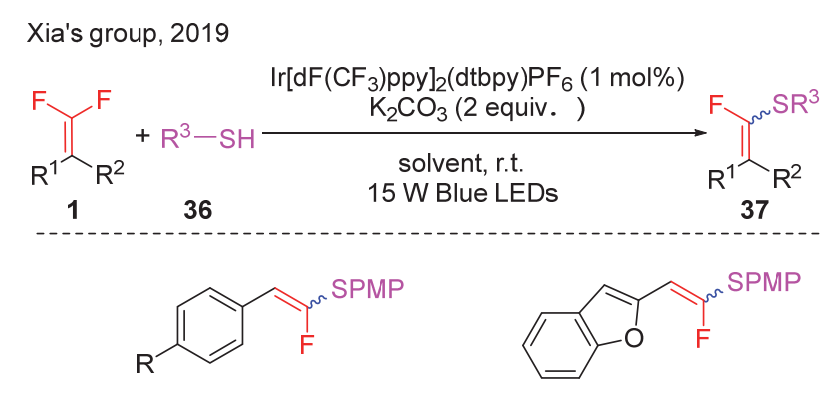

37a, $\mathrm{R}=\mathrm{Ph}, 83 \%(36 \mathrm{~h}), Z: E=40: 60 \quad 37 \mathrm{c}, 72 \%(36 \mathrm{~h}), Z: E=22: 78$ $37 \mathrm{~b}, \mathrm{R}=\mathrm{OCF}_{2} \mathrm{H}, 93 \%(36 \mathrm{~h}), \mathrm{Z:E}=26: 74$<smiles>[R]C(SC(F)=C(c1ccccc1)c1ccccc1)C(=O)OC</smiles>

$37 \mathrm{~d}, \mathrm{R}=\mathrm{H}, 86 \%(24 \mathrm{~h})$ $37 \mathrm{e}, \mathrm{R}=\mathrm{Me}, 95 \%(18 \mathrm{~h})$

图式 25 可见光催化偕二氟烯烃与硫醇的脱氟交叉偶联反应 Scheme 25 Visible-light catalyzed defluorinative cross-coupling of gem-difluoroalkenes with thiols

强氧化性的激发态 $\operatorname{Ir} *(\mathrm{III})$ 物种将硫负离子 $\mathbf{A}$ 氧化 为硫自由基 $\mathbf{B}$, 其自身被还原为 $\mathrm{Ir}(\mathrm{II})$ 物种, 随后硫自由 基 $\mathbf{B}$ 对烯烃 $\mathbf{1}$ 加成生到苠基自由基 $\mathbf{C}$, 并被 $\operatorname{Ir}(\mathrm{II})$ 物种还 原为碳负离子 $\mathbf{D}$, 随后脱除氟负离子得到目标产物, $\operatorname{Ir}(\mathrm{II})$ 物种则被氧化为基态 $\operatorname{Ir}(\mathrm{III})$ 完成催化循环(Scheme 26).

2021 年, 史大永等 ${ }^{[52]}$ 研究了可见光促进偕二氟烯 烃与芳基亚磺酸钠 38 的脱氧脱氟自由基偶联反应, 制 备了一系列 $\alpha$-氟- $\beta$-芳基烯基硫醚衍生物 39 (Scheme $27)$. 反应以 $\mathrm{Na}_{2}(\operatorname{Eosin} \mathrm{Y})$ 为光敏剂、三苯基膦为还原剂,

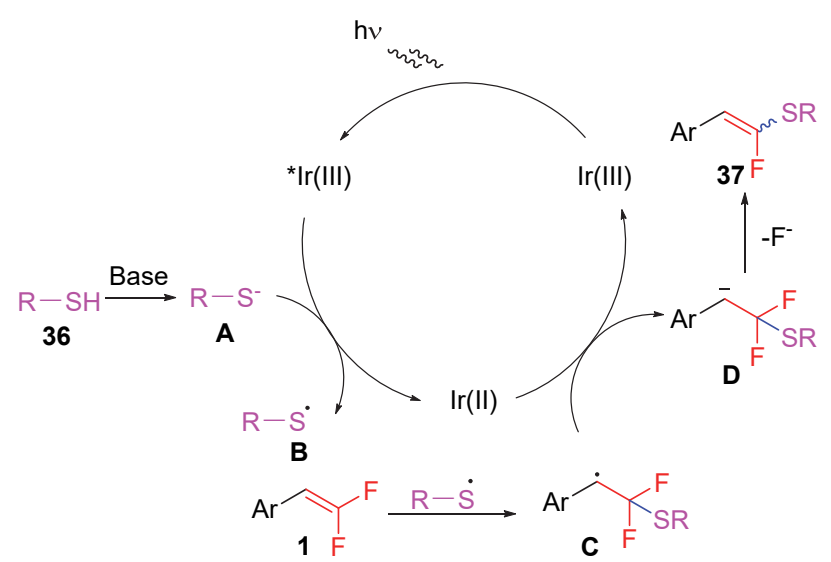

图式 26 偕二氟烯烃与硫醇的脱氟交叉偶联反应机理

Scheme 26 Reaction mechanism for the defluorinative crosscoupling of gem-difluoroalkenes with thiols

反应条件温和，具有良好的官能团容忍性和底物适用 性，菜基亚磺酸钠、噻吩基亚磺酸钠及甲氧基、三氟甲 基、氰基、酯基、卤素原子等取代的苯基亚磺酸钠，以 及各类单取代、双取代偕二氟烯烃等底物都能利用该策 略以中等至优异的收率和良好的立体选择性制备相应 的烯基硫醚产物.
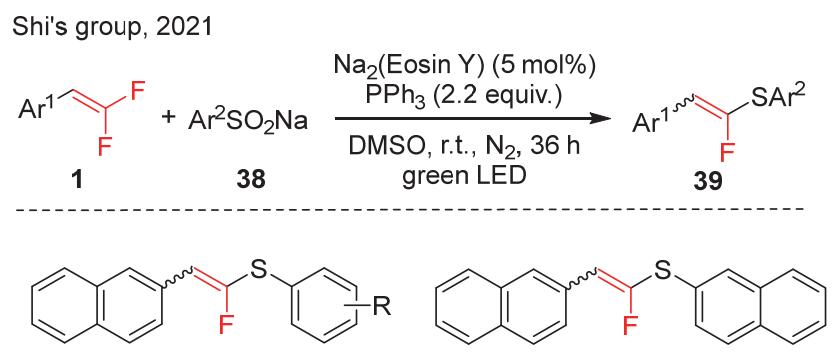

39a, $\mathrm{R}=\mathrm{H}, 94 \%, E: Z=92.5: 7.5$

39b, $\mathrm{R}=4-\mathrm{Me}, 81 \%, E: Z=92: 8$

$39 \mathrm{c}, \mathrm{R}=4-\mathrm{CF}_{3}, 50 \%, E: Z=92: 8$

$39 \mathrm{~d}, \mathrm{R}=4-\mathrm{CO}_{2} \mathrm{Me}, 76 \%, E: Z=89: 11$

$39 e, \mathrm{R}=3-\mathrm{CN}, 66 \%, E: Z=84: 16$

39f, $86 \%, E: Z=89: 11$<smiles>FC(=Cc1cc2ccccc2c2ccccc12)Sc1ccc(Cl)cc1</smiles><smiles>FC(=Cc1ccc2ccccc2c1)Sc1cccs1</smiles>

$39 g, 58 \%, E: Z=41: 59$

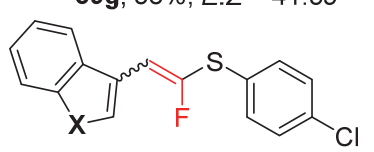

39i, $X=O, 51 \%, E: Z=87.5: 12.5$ 39j, $X=S, 60 \%, E: Z=92: 8$

$39 \mathrm{~h}, 83 \%, E: Z=69: 31$<smiles>CC(COC(C)(C)C)NC(=O)c1ccc(C=C(F)c2ccc(Cl)cc2)cc1</smiles>

图式 27 可见光催化 $\alpha$-氟- $\beta$-芳基烯基硫醚的合成 Scheme 27 Visible-light catalyzed the synthesis of $\alpha$-fluoro$\beta$-arylalkenyl sulfides 
TEMPO 或 1,4-二硝基苯可以完全抑制反应的进行 (Eq. 2), 当以苯硫酚钠为硫源时, 在标准条件下反应也 能以 $89 \%$ 的收率得到硫醚产物, 表明放基负离子可能是 关键中间体(Eq. 3). 推测的反应机理如下(Scheme 28): 苯亚磺酸钠在可见光氧化还原催化体系中, 高效地形成 氧中心自由基 $\mathbf{A}$, 随后与三苯基膦反应形成膦中心自由 基 $\mathbf{B}$, 经 $\beta$-裂解脱除三苯基氧膦得到硫中心自由基 $\mathbf{C}$ 并 异构化为氧中心自由基 $\mathbf{C}^{\prime}, \mathbf{C}^{\prime}$ 进一步与三苯基膦作用脱 除另一分子三苯基氧膦得到苯颈基自由基 $\mathbf{D}$, 该自由基 可以被可见光氧化还原催化剂负离子还原为苯颈基负 离子 $\mathbf{E}$, 并对偕二氟烯烃亲核加成得到碳负离子 $\mathbf{F}$ (Path A); 另外, 苯颈基自由基 $\mathbf{D}$ 也可以直接对偕二氟烯烃加
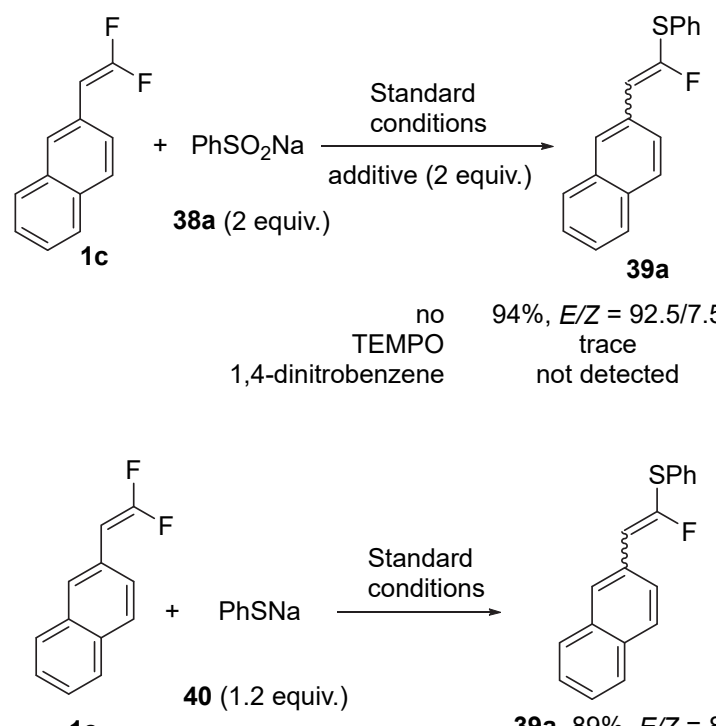

1c

39a, $89 \%, E / Z=85: 15$

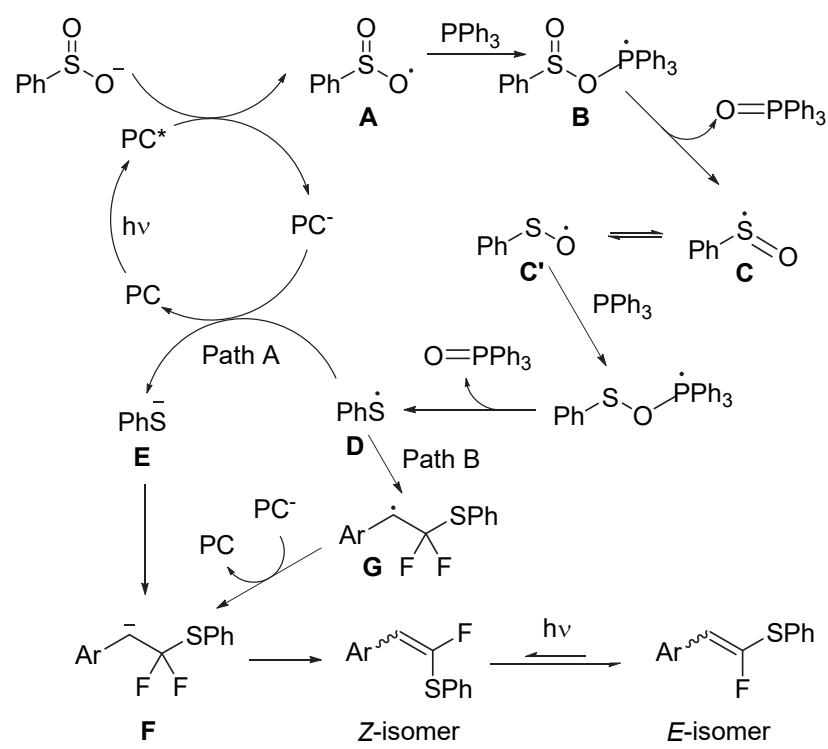

图式 $28 \alpha$-氟- $\beta$-芳基烯基硫醚合成的反应机理 Scheme 28 Reaction mechanism for the synthesis of $\alpha$-fluoro$\beta$-arylalkenyl sulfides
成得到苠基自由基 $\mathbf{G}$, 然后被还原为碳负离子 $\mathbf{F}$ (Path $\mathrm{B})$. 最后, 碳负离子 $\mathbf{F}$ 消除氟负离子得到目标产物. 值 得注意的是, 由于氟和芳环之间的电子排斥作用, 首先 生成的是 $Z$ 式产物, 之后在可见光促进下发生 $Z$ 式到 $E$ 式的异构化.

\section{4 结论与展望}

综述了可见光氧化还原催化、可见光氧化还原/过渡 金属协同催化偕二氟烯烃 $\mathrm{C}-\mathrm{F}$ 键官能化反应的研究进 展, 对反应类型进行了归纳和总结. 可见光催化的偕二 氟烯烃 $\mathrm{C}-\mathrm{F}$ 键官能化反应已经被应用于各类碳-碳及 碳-杂键的高效构建，实现了 $\mathrm{C}-\mathrm{F}$ 键的烷基化、烯基化、 羧基化、三氟甲基化、嗍化及硫醚化等，避免了有机金 属试剂的使用，且反应在室温条件下进行，使得反应更 经济高效和绿色环保，促进了此类反应在有机合成和药 物合成中的应用.

尽管可见光催化的偕二氟烯烃 $\mathrm{C}-\mathrm{F}$ 键官能化反应 取得了快速的发展，该领域仍然存在诸多挑战. 主要包 括: (1)提高反应中各类自由基中间体的反应活性和化学 选择性, 精准调控反应的立体选择性; (2)实现 $\mathrm{C}-\mathrm{O}$ 、 $\mathrm{C}-\mathrm{N} 、 \mathrm{C}-\mathrm{Si} 、 \mathrm{C}-\mathrm{P}$ 等其他类型碳-杂原子化学键的高 效构建; (3)新型廉价易得、环境友好可见光催化体系的 开发及反应机理的深入系统研究. 总体而言, 可见光催 化偕二氟烯烃 $\mathrm{C}-\mathrm{F}$ 键官能化反应的研究领域充满活力, 相信随着可见光化学、自由基化学的蓬勃发展及广大科 研工作者的不断努力, 可见光催化偕二氟烯烃 $\mathrm{C}-\mathrm{F}$ 键 官能化将得到重要发展，并被广泛应用于构筑各类具有 重要应用价值的单氟烯烃化合物.

\section{References}

[1] (a) Johnson, B. M.; Shu, Y.-Z.; Zhuo, X.; Meanwell, N. A. J. Med. Chem. 2020, 63, 6315.

(b) Mei, H.; Han, J.; Fustero, S.; Medio-Simon, M.; Sedgwick, D. M.; Santi, C.; Ruzziconi, R.; Soloshonok, V. A. Chem.-Eur. J. 2019 , 25, 11797.

(c) Liu, H.; Ge, L.; Wang, D.-X.; Chen, N.; Feng, C. Angew. Chem., Int. Ed. 2019, 58, 3918.

(d) Rong, J.; Ni, C.; Wang, Y.; Kuang, C.; Gu, Y.; Hu, J. Acta Chim. Sinica 2017, 75, 105 (in Chinese).

(荣健，倪传法，王云泽，匡翠文，顾玉诚，胡金波，化学学报， 2017, 75, 105.)

(e) Zhou, Y.; Wang, J.; Gu, Z.; Wang, S.; Zhu, W.; Aceña, J. L.; Soloshonok, V. A.; Izawa, K.; Liu, H. Chem. Rev. 2016, 116, 422.

(f) Zhang, K.; Xu, X.-H.; Qing, F.-L. Chin. J. Org. Chem. 2015, 35, 556 (in Chinese).

(张柯, 徐修华, 卿凤翎, 有机化学, 2015, 35, 556.)

(g) Xiao, Y.; Pan, Q.; Zhang, X. Acta Chim. Sinica 2015, 73, 387 (in Chinese).

(肖玉兰, 潘强, 张新刚, 化学学报, 2015, 73, 387.)

(h) Gillis, E. P.; Eastman, K. J.; Hill, M. D.; Donnelly, D. J.; Meanwell, N. A. J. Med. Chem. 2015, 58, 8315.

[2] (a) O'Hagan, D.; Deng, H. Chem. Rev. 2015, 115, 634.

(b) Liang, T.; Neumann, C. N.; Ritter, T. Angew. Chem., Int. Ed. 
2013, 52, 8214.

(c) Purser, S.; Moore, P. R.; Swallow, S.; Gouverneur, V. Chem. Soc. Rev. 2008, 37, 320

(d) Thayer, A. M. Chem. Eng. News 2006, 84, 15.

[3] (a) Sun, H.; Liu, T.; Yu, J.; Lau, T. K.; Zhang, G.; Zhang, Y.; Su, M.; Tang, Y.; Ma, R.; Liu, B.; Liang, J.; Feng, K.; Lu, X.; Guo, X.; Gao, F.; Yan, H. Energy Environ. Sci. 2019, 12, 3328.

(b) Li, X.; Pan, F.; Sun, C.; Zhang, M.; Wang, Z.; Du, J.; Wang, J.; Xiao, M.; Xue, L.; Zhang, Z.-G.; Zhang, C; Liu, F.; Li, Y. Nat. Commun. 2019, 10, 519.

(c) Ge, J.; Fan, L.; Wang, J.; Zhang, Q.; Liu, Z.; Zhang, E.; Liu, Q.; Yu, X.; Lu, B. Adv. Energy Mater. 2018, 8, 1801477.

(d) Ni, C.; Hu, J. Chem. Soc. Rev. 2016, 45, 5441.

(e) Harsanyi, A.; Sandford, G. Org. Process Res. Dev. 2014, 18, 981.

(f) Pagliaro, M.; Ciriminna, R. J. Mater. Chem. 2005, 15, 4981.

(g) Müller, K.; Faeh, C.; Diederich, F. Science 2007, 317, 1881.

[4] (a) Koley, S.; Altman, R. A. Isr. J. Chem. 2020, 60, 313.

(b) Drouin, M.; Hamel, J.-D.; Paquin, J.-F. Synthesis 2018, 50, 881.

(c) Zhang, X.; Cao, S. Tetrahedron Lett. 2017, 58, 375.

(d) Liao, F.; Yu, J.; Zhou, J. Chin. J. Org. Chem. 2017, 37, 2175 (in Chinese).

(廖富民, 余金生, 周剑, 有机化学, 2017, 37, 2175.)

(e) Landelle, G.; Bergeron, M.; Turcotte-Savard, M.; Paquin, J. Chem. Soc. Rev. 2011, 40, 2867.

(f) Yanai, H.; Taguchi, T. Eur. J. Org. Chem. 2011, 5939.

[5] (a) Sommer, H.; Fürstner, A. Chem.-Eur. J. 2017, 23, 558.

(b) Zhao, Y.; Jiang, F.; Hu, J. J. Am. Chem. Soc. 2015, 137, 5199.

(c) Malo-Forest, M.; Landelle, G.; Roy, J.-A.; Lacroix, J.; Gaudreault, R.-C.; Paquin, J.-F. Bioorg. Med. Chem. Lett. 2013, 23, 1712 .

(d) Osada, S.; Sano, S.; Ueyama, M.; Chuman, Y.; Kodama, H.; Sakaguchi, K. Bioorg. Med. Chem. 2010, 18, 605.

(e) Oishi, S.; Kamitani, H.; Kodera, Y.; Watanabe, K.; Kobayashi, K.; Narumi, T.; Tomita, K.; Ohno, H.; Naito, T.; Kodama, E.; Matsuoka, M.; Fujii, N. Org. Biomol. Chem. 2009, 7, 2872.

[6] (a) Drouin, M.; Wadhwani, P.; Grage, S. L.; Burck, J.; Reichert, J.; Tremblay, S.; Mayer, M. S.; Diel, C.; Staub, A.; Paquin, J.-F.; Ulrich, A. S. Chem.-Eur. J. 2020, 26, 1511.

(b) Drouin, M.; Arenas, J. L.; Paquin, J. F. ChemBioChem 2019, 20 , 1817.

(c) Drouin, M.; Paquin, J.-F. Beilstein J. Org. Chem. 2017, 13, 2637.

(d) Zhao, Y.; Jiang, F.; Hu, J. J. Am. Chem. Soc. 2015, 137, 5199.

[7] Macias, F. A.; De Siqueira, J. M.; Chinchilla, N.; Marin, D.; Varela, R. M.; Molinillo, J. M. G. J. Agric. Food Chem. 2006, 54, 9843.

[8] (a) Babudri, F.; Cardone, A.; Farinola, G. M.; Martinelli, C.; Mendichi, R.; Naso, F.; Striccoli, M. Eur. J. Org. Chem. 2008, 1977. (b) Babudri, F.; Farinola, G. M.; Naso, F.; Ragni, R. Chem. Commun. 2007, 1003 .

[9] (a) Guérin, D.; Gaumont, A.-C.; Dez, I.; Mauduit, M.; CouveBonnaire, S.; Pannecoucke, X. ACS Catal. 2014, 4, 2374.

(b) Dai, W.; Xiao, J.; Jin, G.; Wu, J.; Cao, S. J. Org. Chem. 2014, 79, 10537.

(c) Debien, L.; Quiclet-Sire, B.; Zard, S. S. Org. Lett. 2012, 14, 5118.

(d) Wong, O. A.; Shi, Y. J. Org. Chem. 2009, 74, 8377.

(e) Dutheuil, G.; Couve-Bonnaire, S.; Pannecoucke, X. Angew. Chem., Int. Ed. 2007, 46, 1290.

[10] (a) Drouin, M.; Hamel, J.-D.; Paquin, J.-F. Synlett 2016, $27,821$.

(b) Champagne, P. A.; Desroches, J.; Hamel, J.-D.; Vandamme, M.; Paquin, J.-F. Chem. Rev. 2015, 115, 9073.

(c) Couve-Bonnaire, S.; Cahard, D.; Pannecoucke, X. Org. Biomol. Chem. 2007, 5, 1151.

[11] (a) Zhang, H.; Wang, E.; Geng, S.; Liu, Z.; He, Y.; Peng, Q.; Feng, Z. Angew. Chem., Int. Ed. 2021, 60, 10211.

(b) Sakaguchi, H.; Ohashi, M.; Ogoshi, S. Angew. Chem., Int. Ed. 2018, 57, 328. (c) Tan, D.-H.; Lin, E.; Ji, W.-W.; Zeng, Y.-F.; Fan, W.-X.; Li, Q.; Gao, H.; Wang, H. Adv. Synth. Catal. 2018, 360, 1032.

[12] (a) Yan, S.-S.; Wu, D.-S.; Ye, J.-H.; Gong, L.; Zeng, X.; Ran, C.-K.; Gui, Y.-Y.; Li, J.; Yu, D.-G. ACS Catal. 2019, 9, 6987.

(b) Hu, J.; Zhao, Y.; Shi, Z. Nat. Catal. 2018, 1, 860.

(c) Ito, H.; Seo, T.; Kojima, R.; Kubota, K. Chem. Lett. 2018, 47, 1330.

(d) Sakaguchi, H.; Uetake, Y.; Ohashi, M.; Niwa, T.; Ogoshi, S.; Hosoya, T. J. Am. Chem. Soc. 2017, 139, 12855.

(e) Zhang, J.; Dai, W.; Liu, Q.; Cao, S. Org. Lett. 2017, 19, 3283.

(f) Thornbury, R. T.; Toste, F. D. Angew. Chem., Int. Ed. 2016, 55, 11629.

(g) Xiong, Y.; Huang, T.; Ji, X.; Wu, J.; Cao, S. Org. Biomol. Chem. 2015, 13, 7389.

(h) Ohashi, M.; Saijo, H.; Shibata, M.; Ogoshi, S. Eur. J. Org. Chem. 2013, 443.

[13] (a) Dai, W.; Shi, H.; Zhao, X.; Cao, S. Org. Lett. 2016, 18, 4284.

(b) Dai, W.; Zhang, X.; Zhang, J.; Lin, Y.; Cao, S. Adv. Synth. Catal. 2016, 358, 183 .

(c) Dai, W.; Xiao, J.; Jin, G.; Wu, J.; Cao, S. J. Org. Chem. 2014, 79, 10537.

(d) Yamada, S.; Shimoji, K.; Takahashi, T.; Konno, T.; Ishihara, T. Chem.-Asian J. 2010, 5, 1846.

(e) Yamada, S.; Noma, M.; Hondo, K.; Konno, T.; Ishihara, T. J. Org. Chem. 2008, 73, 522.

(f) Yamada, S.; Noma, M.; Konno, T.; Ishihara, T.; Yamanaka, H. Org. Lett. 2006, 8, 743.

[14] (a) Zhang, J.; Wang, B.; Liu, S.; Cao, S. Chin. J. Org. Chem. 2019, 39, 249 (in Chinese).

(张娟, 王碧云, 刘熠森, 曹松, 有机化学, 2019, 39, 249.)

(b) Ohashi, M.; Kambara, T.; Hatanaka, T.; Saijo, H.; Doi, R.; Ogoshi, S. J. Am. Chem. Soc. 2011, 133, 3256.

(c) Takachi, M.; Kita, Y.; Tobisu, M.; Fukumoto, Y.; Chatani, N. Angew. Chem., Int. Ed. 2010, 49, 8717.

(d) Saeki, T.; Takashima, Y.; Tamao, K. Synlett 2005, 1771.

[15] Wu, F.-P.; Yuan, Y.; Liu, J.; Wu, X.-F. Angew. Chem., Int. Ed. 2021, 60, 8818.

[16] Ma, Q.; Wang, Y.; Tsui, G. C. Angew. Chem., Int. Ed. 2020, 59, 11293.

[17] (a) Song, S.; Liu, H.; Wang, L.; Zhu, L.; Loh, T.-P.; Feng, C. Chin. J. Chem. 2019, 37, 1036.

(b) Zhou, L.; Zhu, C.; Loh, T.-P.; Feng, C. Chem. Commun. 2018, 54,5618 .

(c) Tian, M.; Yang, X.; Zhang, B.; Liu, B.; Li, X. Org. Chem. Front. 2018, 5, 3406.

(d) Li, N.; Chang, J.; Kong, L.; Li, X. Org. Chem. Front. 2018, 5, 1978.

(e) Gong, T.-J.; Xu, M.-Y.; Yu, S.-H.; Yu, C.-G.; Su, W.; Lu, X.; Xiao, B.; Fu, Y. Org. Lett. 2018, 20, 570.

(f) Murakami, N.; Yoshida, M.; Yoshino, T.; Matsunaga, S. Chem. Pharm. Bull. 2018, 6, 51.

(g) Wu, J.-Q.; Zhang, S.-S.; Gao, H.; Qi, Z.; Zhou, C.-J.; Ji, W.-W.; Liu, Y.; Chen, Y.; Li, Q.; Li, X.; Wang, H. J. Am. Chem. Soc. 2017, 139, 3537.

(h) Zell, D.; Dhawa, U.; Müller, V.; Bursch, M.; Grimme, S.; Ackermann, L. ACS Catal. 2017, 7, 4209.

(i) Cai, S.-H.; Ye, L.; Wang, D.-X.; Wang, Y.-Q.; Lai, L.-J.; Zhu, C.; Feng, C.; Loh, T.-P. Chem. Commun. 2017, 53, 8731.

(j) Kong, L.; Liu, B.; Zhou, X.; Wang, F.; Li, X. Chem. Commun. 2017, 53, 10326 .

(k) Zell, D.; Meller, V.; Dhawa, U.; Bursch, M.; Presa, R. R.; Grimme, S.; Ackermann, L. Chem.-Eur. J. 2017, 23, 12145.

(1) Tian, P.; Feng, C.; Loh, T.-P. Nat. Commun. 2015, 6, 7472.

[18] (a) Amii, H.; Uneyama, K. Chem. Rev. 2009, 109, 2119.

(b) Ichikawa, J. Chim. Oggi 2007, 25, 54.

[19] (a) Cannalire, R.; Pelliccia, S.; Sancineto, L.; Novellino, E.; Tron, G. C.; Giustiniano, M. Chem. Soc. Rev. 2021, 50, 766.

(b) Yu, X.-Y.; Chen, J.-R.; Xiao, W.-J. Chem. Rev. 2021, 121, 506. 
(c) Li, Z.; Jin, J.; Huang, S. Chin. J. Org. Chem. 2020, 40, 563 (in Chinese).

(李祯龙，金健，黄莎华，有机化学, 2020, 40, 563.)

(d) Zhou, Q.-Q.; Zou, Y.-Q.; Lu, L.-Q.; Xiao, W.-J. Angew. Chem., Int. Ed. 2019, 58, 1586.

(e) Kong, Y.; Xu, W.; Ye, F.; Weng, J. Chin. J. Org. Chem. 2019, 39, 3065 (in Chinese)

(孔瑶蕾, 徐雯秀, 叶飞霞, 翁建全, 有机化学, 2019, 39, 3065.)

(f) Parasram, M.; Gevorgyan, V. Chem. Soc. Rev. 2017, 46, 6227.

[20] (a) Leifert, D.; Studer, A. Angew. Chem., Int. Ed. 2020, 59, 74.

(b) Wang, S.; Tang, S.; Lei, A. Sci. Bull. 2018, 63, 1006.

(c) Yi, H.; Zhang, G.; Wang, H.; Huang, Z.; Wang, J.; Singh, A. K.; Lei, A. Chem. Rev. 2017, 117, 9016.

(d) Xie, J.; Jin, H.; Hashmi, A. S. K. Chem. Soc. Rev. 2017, 46, 5193.

(e) Togo, H. Advanced Free Radical Reactions for Organic Synthesis, Elsevier, Amsterdam, 2004, pp. 1 2.

[21] (a) Nobile, E.; Castanheiro, T.; Besset, T. Angew. Chem., Int. Ed. 2021, 60, 12170.

(b) Wu, X.; Zhu, C. Acc. Chem. Res. 2020, 53, 1620.

[22] Xie, J.; Yu, J.; Rudolph, M.; Rominger, F.; Hashmi, A. S. K. Angew. Chem., Int. Ed. 2016, 55, 9416.

[23] (a) Wilkinson, B.; Zhu, M.; Priestley, N. D.; Nguyen, H. H. T.; Morimoto, H.; Williams, P. G.; Chan, S. I.; Floss, H. G. J. Am. Chem. Soc. 1996, 118, 921.

(b) Step, E. N.; Turro, N. J. J. Photochem. Photobiol. A 1994, 84, 249

(c) Scaiano, J. C. J. Phys. Chem. 1981, 85, 2851.

(d) Livant, P.; Lawler, R. G. J. Am. Chem. Soc. 1976, 98, 6044.

[24] Tian, H.; Xia, Q.; Wang, Q.; Dong, J.; Liu, Y.; Wang, Q. Org. Lett. 2019, 21,4585 .

[25] (a) Zuo, Z.; Ahneman, D. T.; Chu, L.; Terrett, J. A.; Doyle, A. G.; MacMillan, D. W. C. Science 2014, 345, 437.

(b) Lowry, M. S.; Goldsmith, J. I.; Slinker, J. D.; Rohl, R.; Pascal, R. A.; Malliaras, G. G.; Bernhard, S. Chem. Mater. 2005, 17, 5712.

[26] Jeffrey, J. L.; Terrett, J. A.; MacMillan, D. W. C. Science 2015, 349, 1532 .

[27] Xie, J.; Yu, J.; Rudolph, M.; Rominger, F.; Hashmi, A. S. K. Angew. Chem., Int. Ed. 2016, 55, 9416.

[28] (a) Chen, H.; Liu, Y. A.; Liao, X. Synthesis 2021, 53, 1.

(b) McMurray, L.; McGuire, T. M.; Howells, R. L. Synthesis 2020, 52, 1719.

(c) Zhou, M.; Qin, P.; Jing, L.; Sun, J.; Du, H. Chin. J. Org. Chem. 2020, 40, 598 (in Chinese).

(周明东, 覃丕涛, 经理珂, 孙京, 杜海武, 有机化学, 2020, 40, 598.)

(d) Liu, J.-Q.; Shatskiy, A.; Matsuura, B. S.; Kärkäs, M. D. Synthesis 2019, 51, 2759.

(e) Rahman, M.; Mukherjee, A.; Kovalev, I. S.; Kopchuk, D. S.; Zyryanov, G. V.; Tsurkan, M. V.; Majee, A.; Ranu, B. C.; Charushin, V. N.; Chupakhin, O. N.; Santra, S. Adv. Synth. Catal. 2019, 361, 2161 .

(f) Nakajima, K.; Miyake, Y.; Nishibayashi, Y. Acc. Chem. Res. 2016, 49, 1946.

(g) Xuan, J.; Zhang, Z.-G.; Xiao, W.-J. Angew. Chem., Int. Ed. 2015, 54, 15632.

[29] Li, J.; Lefebvre, Q.; Yang, H.; Zhao, Y.; Fu, H. Chem. Commun. 2017, 53, 10299.

[30] Yang, H.; Tian, C.; Qiu, D.; Tian, H.; An, G.; Li, G. Org. Chem. Front. 2019, 6, 2365.

[31] (a) Xu, W.; Ma, J.; Yuan, X.; Dai, J.; Xie, J.; Zhu, C. Angew. Chem., Int. Ed. 2018, 57, 10357.

(b) Luo, J.; Zhang, J. ACS Catal. 2016, 6, 873

[32] Galicia, M.; Gonzalez, F. J. J. Electrochem. Soc. 2002, 149, D46.

[33] (a) Ye, S.; Wu, J. Acta Chim. Sinica 2019, 77, 814 (in Chinese). (叶盛青, 吴款, 化学学报, 2019, 77, 814.)

(b) Wang, P.-Z.; Chen, J.-R.; Xiao, W.-J. Org. Biomol. Chem. 2019, 17,6936 . (c) Milligan, J. A.; Phelan, J. P.; Badir, S. O.; Molander, G. A. Angew. Chem., Int. Ed. 2019, 58, 6152.

(d) Huang, W.; Cheng, X. Synlett 2017, 28, 148.

[34] Du, H.-W.; Sun, J.; Gao, Q.-S.; Wang, J.-Y.; Wang, H.; Xu, Z.; Zhou, M.-D. Org. Lett. 2020, 22, 1542.

[35] Lemos, A.; Lemaire, C.; Luxen, A. Adv. Synth. Catal. 2019, 361, 1500.

[36] Wang, P.; Chen, J.; Xiao, W. Org. Biomol. Chem. 2019, 17, 6936.

[37] (a) Yao, H.; Hu, W.; Zhang, W. Molecules 2021, 26, 105.

(b) Pang, J.; Wu, J.; Wu, F. Chin. J. Org. Chem. 2021, 41, 983 (in Chinese).

(潘军, 吴晶晶, 吴范宏, 有机化学, 2021, 41, 983.)

(c) Wu, Y.-C.; Xiao, Y.-T.; Yang, Y.-Z.; Song, R.-J.; Li, J.-H. Chem CatChem 2020, 12, 5312.

(d) Wu, X.; Zhu, C. Acc. Chem. Res. 2020, 53, 1620.

(e) Diccianni, J.; Lin, Q.; Diao, T. Acc. Chem. Res. 2020, 53, 906

(f) Jiang, H.; Studer, A. Chem. Soc. Rev. 2020, 49, 1790.

(g) Li, Z.-L.; Fang, G.-C.; Gu, Q.-S.; Liu, X.-Y. Chem. Soc. Rev. 2020, 49, 32

(h) Bao, X.; Li, J.; Jiang, W.; Huo, C. Synthesis 2019, 51, 4507.

(i) Kawamura, S.; Sodeoka, M. Bull. Chem. Soc. Jpn. 2019, 92, 1245 .

(j) Wang, X.; Studer, A. Acc. Chem. Res. 2017, 50, 1712.

[38] Wang, Q.; Qu, Y.; Tian, H.; Liu, Y.; Song, H.; Wang, Q. Chem.-Eur. J. 2019, 25, 8686.

[39] (a) Chen, L.; Francis, H.; Carrow, B. P. ACS Catal. 2018, 8, 2989.

(b) Bulfield, D.; Huber, S. M. J. Org. Chem. 2017, 82, 13188.

(c) Chen, L.; Sanchez, D. R.; Zhang, B.; Carrow, B. P. J. Am. Chem. Soc. 2017, 139, 12418.

(d) Handa, S.; Wang, Y.; Gallou, F.; Lipshutz, B. H. Science 2015, 349, 1087.

(e) Kinzel, T.; Zhang, Y.; Buchwald, S. L. J. Am. Chem. Soc. 2010 , 132, 14073 .

(f) Miyaura, N.; Suzuki, A. Chem. Rev. 1995, 95, 2457.

[40] (a) Gao, P.; Yuan, C.; Zhao, Y.; Shi, Z. Chem 2018, 4, 2201.

(b) Kojima, R.; Akiyama, S.; Ito, H. Angew. Chem., Int. Ed. 2018, 57, 7196 .

(c) Lim, S.; Song, D.; Jeon, S.; Kim, Y.; Kim, H.; Lee, S.; Cho, H.; Lee, B. C.; Kim, S. E.; Kim, K.; Lee, E. Org. Lett. 2018, $20,7249$.

(d) Sakaguchi, H.; Uetake, Y.; Ohashi, M.; Niwa, T.; Ogoshi, S.; Hsoya, T. J. Am. Chem. Soc. 2017, 139, 12855.

(e) Liu, Y.; Zhou, Y.; Zhao, Y.; Qu, J. Org. Lett. 2017, 19, 946.

(f) Zhou, J.; Kuntze-Fechner, M. W.; Bertermann, R.; Paul, U. S.

D.; Berthel, J. H. J.; Friedrich, A.; Du, Z.; Marder, T. B.; Radius, U. J. Am. Chem. Soc. 2016, 138, 5250.

(g) Guo, W.-H.; Min, Q.-Q.; Gu, J.-W.; Zhang, X. Angew. Chem., Int. Ed. 2015, 54, 9075.

[41] Xu, W.; Jiang, H.; Leng, J.; Ong, H.-W.; Wu, J. Angew. Chem., Int. Ed. 2020, 59, 4009.

[42] (a) Zhu, C.; Yue, H.; Chu, L.; Rueping, M. Chem. Sci. 2020, 11, 4051.

(b) Abreu, M. D.; Belmont, P.; Brachet, E. Eur. J. Org. Chem. 2020, $2020,1327$.

(c) Zhang, H.-H.; Yu, S. Acta Chim. Sinica 2019, 77, 832 (in Chinese).

(张洪浩, 俞寿云, 化学学报, 2019, 77, 832.)

(d) Wang, C.-S.; Dixneuf, P. H.; Soulé, J.-F. Chem. Rev. 2018, 118, 7532 .

(e) Zhou, W.-J.; Zhang, Y.-H.; Gui, Y.-Y.; Sun, L.; Yu, D.-G. Synthesis 2018, 50, 3359.

(f) Twilton, J.; Le, C.; Zhang, P.; Shaw, M. H.; Evans, R. W.; MacMillan, D. W. C. Nat. Rev. 2017, 1, 0052.

(g) Ruan, L.; Dong, Z.; Chen, C.; Wu, S.; Sun, J. Chin. J. Org. Chem. 2017, 37, 2544 (in Chinese).

(阮利衡, 董振诚, 陈春欣, 吴爽, 孙京, 有机化学, 2017, 37, 2544.)

(h) Skubi, K. L.; Blum, T. R.; Yoon, T. P. Chem. Rev. 2016, 116, 10035 . 
(i) Tellis, J. C.; Kelly, C. B.; Primer, D. N.; Jouffroy, M.; Patel, N. R.; Molander, G. A. Acc. Chem. Res. 2016, 49, 1429.

[43] Zhu, C.; Zhang, Y.-F.; Liu, Z.-Y.; Zhou, L.; Liu, H.; Feng, C. Chem. Sci. 2019, 10, 6721.

[44] (a) Li, J.; Luo, Y.; Cheo, H. W.; Lan, Y.; Wu, J. Chem 2019, 5, 192. (b) Matsui, J. K.; Gutiérrez-Bonet, Á.; Rotella, M.; Alam, R.; Gutierrez, O.; Molander, G. A. Angew. Chem., Int. Ed. 2018, 57, 15847.

(c) Meng, Q.-Y.; Wang, S.; Huff, G. S.; König, B. J. Am. Chem. Soc. 2018, 140, 3198.

(d) Tellis, J. C.; Kelly, C. B.; Primer, D. N.; Jouffroy, M.; Patel, N. R.; Molander, G. A. Acc. Chem. Res. 2016, 49, 1429.

[45] (a) Chuentragool, P.; Yadagiri, D.; Morita, T.; Sarkar, S.; Parasram, M.; Wang, Y.; Gevorgyan, V. Angew. Chem., Int. Ed. 2019, 58, 1794.

(b) Ratushnyy, M.; Parasram, M.; Wang, Y.; Gevorgyan, V. Angew. Chem., Int. Ed. 2018, 57, 2712.

(c) Zhou, W.-J.; Cao, G.-M.; Shen, G.; Zhu, X.-Y.; Gui, Y.-Y.; Ye, J.-H.; Sun, L.; Liao, L.-L.; Li, J.; Yu, D.-G. Angew. Chem., Int. Ed. 2017, 56, 15683.

(d) Parasram, M.; Gevorgyan, V. Chem. Soc. Rev. 2017, 46, 6227.

[46] Shimomaki, K.; Murata, K.; Martin, R.; Iwasawa, N. J. Am. Chem. Soc. 2017, 139, 9467.

[47] Wu, L.-H.; Cheng, J.-K.; Shen, L.; Shen, Z.-L.; Loh, T. P. $A d v$.
Synth. Catal. 2018, 360, 3894.

[48] Nenajdenko, V. G.; Korotchenko, V. N.; Shastin, A. V.; Balenkova, E. S. Russ. Chem. Bull. 2004, 53, 1034.

[49] (a) Boyd, D. A. Angew. Chem., Int. Ed. 2016, 55, 15486.

(b) Feng, M.; Tang, B.; Liang, S.; Jiang, X. Curr. Top. Med. Chem. 2016, 16, 1200.

(c) Ilardi, E. A.; Vitaku, E.; Njardarson, J. T. J. Med. Chem. 2014, $57,2832$.

[50] (a) Wang, B.; Zhou, Y.; Luo, S.; Luo, X.; Chen, W.; Yang, S.; Wang, Z. Chin. J. Org. Chem. 2021, 41, 171 (in Chinese).

(王柏文, 周永军, 罗时荷, 罗晓燕, 陈伟清, 杨诗敏, 汪朝阳, 有机化学, 2021, 41, 171.)

(b) Lou, J.; Wang, Q.; Wu, P.; Wang, H.; Zhou, Y.-G.; Yu, Z. Chem. Soc. Rev. 2020, 49, 4307.

(c) Yang, W.; Zhang, M.; Chen, W.; Yang, X.; Feng, J. Chin. J. Org. Chem. 2020, 40, 4060 (in Chinese).

(杨文超, 张明明, 陈旺, 杨小虎, 冯建国, 有机化学, 2020, 40, 4060.)

(d) Kaiser, D.; Klose, I.; Oost, R.; Neuhaus, J.; Maulide, N. Chem. Rev. 2019, 119, 8701.

(e) Wang, L.; He, W.; Yu, Z. Chem. Soc. Rev. 2013, 42, 599.

[51] Wang, J.; Huang, B.; Yang, C.; Xia, W. Chem. Commun. 2019, 55, 11103.

[52] Li, Y.; Li, X.; Li, X.; Shi, D. Chem. Commun. 2021, 57, 2152.

(Zhao, C.) 